\title{
Energy Quantization for Willmore Surfaces and Applications
}

\author{
Yann Bernard* and Tristan Rivière ${ }^{\dagger}$
}

\begin{abstract}
We prove a bubble-neck decomposition together with an energy quantization result for sequences of Willmore surfaces into $\mathbb{R}^{m}$ with uniformly bounded energy and non-degenerating conformal type. We deduce the strong compactness of Willmore closed surfaces of a given genus modulo the Möbius group action, below some energy threshold.
\end{abstract}

Math. Class. 30C70, 58E15, 58E30, 49Q10, 53A30, 35R01, 35J35, 35J48, 35J50.

\section{Introduction}

Let $\vec{\Phi}$ be an immersion from a closed abstract two-dimensional manifold $\Sigma$ into $\mathbb{R}^{m \geq 3}$. We denote by $g:=\vec{\Phi}^{*} g_{\mathbb{R}^{m}}$ the pull back by $\vec{\Phi}$ of the flat canonical metric $g_{\mathbb{R}^{m}}$ of $\mathbb{R}^{m}$, also called the first fundamental form of $\vec{\Phi}$, and we let $d v l_{g}$ be its associated volume form. The Gauss map of the immersion $\vec{\Phi}$ is the map taking values in the Grassmannian of oriented $m-2$-planes in $\mathbb{R}^{m}$ given by

$$
\vec{n}_{\vec{\Phi}}:=\star \frac{\partial_{x_{1}} \vec{\Phi} \wedge \partial_{x_{2}} \vec{\Phi}}{\left|\partial_{x_{1}} \vec{\Phi} \wedge \partial_{x_{2}} \vec{\Phi}\right|},
$$

where $\star$ is the usual Hodge star operator in the Euclidean metric.

Denoting by $\pi_{\vec{n}_{\vec{\Phi}}}$ the orthonormal projection of vectors in $\mathbb{R}^{m}$ onto the $m-2$-plane given by $\vec{n}_{\vec{\Phi}}$, the second fundamental form may be expressed a: 1

$$
\forall X, Y \in T_{p} \Sigma \quad \overrightarrow{\mathbb{I}}_{p}(X, Y):=\pi_{\vec{n}_{\vec{\Phi}}} d^{2} \vec{\Phi}(X, Y)
$$

The mean curvature vector of the immersion at $p$ is

$$
\vec{H}_{\vec{\Phi}}:=\frac{1}{2} \operatorname{tr}_{g}(\overrightarrow{\mathbb{I}})=\frac{1}{2}\left[\overrightarrow{\mathbb{I}}\left(\varepsilon_{1}, \varepsilon_{1}\right)+\overrightarrow{\mathbb{I}}\left(\varepsilon_{2}, \varepsilon_{2}\right)\right],
$$

where $\left(\varepsilon_{1}, \varepsilon_{2}\right)$ is an orthonormal basis of $T_{p} \Sigma$ for the metric $g_{\vec{\Phi}}$.

In the present paper, we study the Lagrangian given by the $L^{2}$-norm of the second fundamental form:

$$
E(\vec{\Phi}):=\int_{\Sigma}|\overrightarrow{\mathbb{I}}|_{g_{\vec{\Phi}}}^{2} d v o l_{g_{\vec{\Phi}}}
$$

An elementary computation gives

$$
E(\vec{\Phi}):=\int_{\Sigma}|\overrightarrow{\mathbb{I}}|_{g_{\vec{\Phi}}}^{2} d v o l_{g_{\vec{\Phi}}}=\int_{\Sigma}\left|d \vec{n}_{\vec{\Phi}}\right|_{g_{\vec{\Phi}}}^{2} d v o l_{g_{\vec{\Phi}}} .
$$

\footnotetext{
${ }^{*}$ Mathematisches Institut, Albert-Ludwigs-Universität, 79004 Freiburg, Germany. Supported by the DFG SFB 71 fund (project B3).

${ }^{\dagger}$ Department of Mathematics, ETH Zentrum, CH-8093 Zürich, Switzerland.

${ }^{1}$ In order to define $d^{2} \vec{\Phi}(X, Y)$ one has to extend locally around $T_{p} \Sigma$ the vector-fields $X$ and $Y$. It is not difficult to check that $\pi_{\vec{n}} d^{2} \vec{\Phi}(X, Y)$ is independent of this extension.
} 
The energy $E$ may accordingly be seen as the Dirichlet Energy of the Gauss map $\vec{n}_{\vec{\Phi}}$ with respect to the induced metric $g_{\vec{\Phi}}$. The Gauss Bonnet theorem implies that

$$
E(\vec{\Phi}):=\int_{\Sigma}|\overrightarrow{\mathbb{I}}|_{g_{\vec{\Phi}}}^{2} d v o l_{g_{\vec{\Phi}}}=4 \int_{\Sigma}\left|\vec{H}_{\vec{\Phi}}\right|^{2} d v o l_{g_{\vec{\Phi}}}-2 \int_{\Sigma} K_{\vec{\Phi}} d v o l_{g_{\vec{\Phi}}}=4 \int_{\Sigma}\left|\vec{H}_{\vec{\Phi}}\right|^{2} d v o l_{g}-4 \pi \chi(\Sigma),
$$

where $K_{\vec{\Phi}}$ is the Gauss curvature of the immersion, and $\chi(\Sigma)$ is the Euler characteristic of the surface $\Sigma$. The energy

is called Willmore energy.

$$
W(\vec{\Phi}):=\int_{\Sigma}\left|\vec{H}_{\vec{\Phi}}\right|^{2} d v o l_{g_{\vec{\Phi}}}
$$

Critical points of the Willmore energy, comprising for example minimal surface 2 , are called Willmore surfaces. Although already known in the XIXth century in the context of the elasticity theory of plates, it was first considered in conformal geometry by Blaschke in Bla3 who sought to merge the theory of minimal surfaces and the conformal invariance property. This Lagrangian has indeed both desired features : its critical points contain minimal surfaces, and it is conformal invariant, owing to the following pointwise identity which holds for an arbitrary immersion $\vec{\Phi}$ of $\Sigma$ into $\mathbb{R}^{m}$ and at every point of $\Sigma$ :

$$
\forall \Xi \quad \text { conformal diffeo. of } \mathbb{R}^{m} \cup\{\infty\} \quad\left[\left|\vec{H}_{\Xi \circ \vec{\Phi}}\right|^{2}-K_{\Xi \circ \vec{\Phi}}\right] d v o l_{g_{\Xi \circ \vec{\Phi}}}=\left[\left|\vec{H}_{\vec{\Phi}}\right|^{2}-K_{\vec{\Phi}}\right] d v o l_{g_{\vec{\Phi}}} .
$$

Using again Gauss Bonnet theorem, the latter implies the conformal invariance of $W$ :

$$
\forall \Xi \quad \text { conformal diffeo. from } \mathbb{R}^{m} \cup\{\infty\} \quad W(\Xi \circ \vec{\Phi})=W(\vec{\Phi})
$$

This conformal invariance implies that the image of a Willmore immersion by a conformal transformation of $\mathbb{R}^{m}$ is still a Willmore immersion. Starting for example from a minimal surface, one may then generate many new Willmore surfaces, simply by applying conformal transformations (naturally, these surfaces need no longer be minimal). In his time, Blaschke used the term conformal minimal for the critical points of $W$, seeking to insist on this idea of merging together the theory of minimal surface with conformal invariance.

An important task in the analysis of Willmore surfaces is to understand the closure of the space of Willmore immersions under a certain level of energy. Because of the non-compactness of the conformal group of transformation of $\mathbb{R}^{m}$, one cannot expect that the space of Wilmore immersions in closed in the strong $C^{l}$-topology. However, locally, in isothermic coordinates 3 , under some universal energy threshold, if the conformal factor of the induced metric $g_{\vec{\Phi}}$ is controlled in $L^{\infty}$, then the immersion is uniformly bounded in any $C^{l}$ norm. More precisely there holds the following $\varepsilon$-regularity result.

Theorem I.1 [Ri2] There exists $\varepsilon(m)>0$ such that, for any Willmore conformal immersion $\vec{\Phi}$ from $B_{1}(0)$ into $\mathbb{R}^{m}$ satisfying

$$
\int_{B_{1}(0)}\left|\nabla \vec{n}_{\vec{\Phi}}\right|^{2} d x<\varepsilon(m)
$$

then for any $l \in \mathbb{N}^{*}$ we have

$$
\left\|e^{-\lambda} \nabla^{l} \vec{\Phi}\right\|_{L^{\infty}\left(B_{1 / 2}\right)} \leq C_{l}\left[\int_{B_{1}(0)}\left|\nabla \vec{n}_{\vec{\Phi}}\right|^{2} d x+1\right]^{1 / 2}
$$

where $C_{l}$ only depends on $l$, while $\lambda$ denotes the conformal parameter of $\vec{\Phi}$. Namely, $\lambda=\left\|\log \left|\partial_{x_{1}} \vec{\Phi}\right|\right\|_{L^{\infty}\left(B_{1}\right)}=$ $\left\|\log \left|\partial_{x_{2}} \vec{\Phi}\right|\right\|_{L^{\infty}\left(B_{1}\right)}$.

\footnotetext{
${ }^{2}$ minimal surfaces satisfy $\vec{H}=0$ and are hence absolute minimizers of $W$.

${ }^{3}$ Analogously to other gauge-invariant problems, such as in Yang-Mills theory, isothermic coordinates or conformal parametrizations provide the optimal symmetry breaking method in the search of pertinent estimates. A detailed discussion on this topic is available in Ri1].
} 
This theorem leads to the concentration of compactness "dialectic" developed by Sacks and Uhlenbeck. In a conformal parametrization, assuming that the conformal factor is $L^{\infty}$-controlled in some subdomain of $\Sigma$, then a sequence of Willmore immersions can fail to convergence strongly in $C^{l}$ only at isolated points ; namely, at those points where the $W^{1,2}$-norm of the Gaus map concentrates. Assuming their induced metric generates a sequence of conformal classes which remains within a compact subdomain of the moduli space of $\Sigma$, the control of the conformal factor of a sequence of conformal immersions with uniformly bounded Willmore energy is also guaranteed, except at those isolated points of $\Sigma$ where the $W^{1,2}$-norm of the Gaus map concentrates. This fact is established in Ri3] (see also proposition III.1 below), and it utlimately follows from the works of Toro [To], of Müller and Sverak [MS], and from the work of Hélein Hel] on immersions with totally bounded curvature.

To fully understand the loss of strong compactness of a sequence of Willmore surfaces, the difficulty is of course to perform the necessary analysis at the "blow up points". This is the aim of the present paper. Under the assumption that the sequence of conformal classes associated to the sequence of Willmore surfaces remains within a compact subdomain of the Moduli space, we show that, modulo extraction of a subsequence, the parametrizing abstract surface splits into three distinct regions : the main region where strong convergence holds, the concentrating parametrization of non-trivial Willmore spheres, and finally the bubble and neck regions connecting the two previous ones and in which we show the energy vanishes.

We now state our main result.

Theorem I.2 Let $\vec{\Phi}_{k}$ be a sequence of Willmore immersions of a closed surface $\Sigma$. Assume that

$$
\limsup _{k \rightarrow+\infty} W\left(\vec{\Phi}_{k}\right)<+\infty
$$

that the conformal class of $\vec{\Phi}_{k}^{*} g_{\mathbb{R}^{m}}$ remains within a compact subdomain of the moduli space of $\Sigma$. Then, modulo extraction of a subsequence, the following energy identity holds

$$
\lim _{k \rightarrow+\infty} W\left(\vec{\Phi}_{k}\right)=W\left(\vec{\xi}_{\infty}\right)+\sum_{s=1}^{p} W\left(\vec{\eta}_{s}\right)+\sum_{t=1}^{q}\left[W\left(\vec{\zeta}_{t}\right)-4 \pi \theta_{t}\right]
$$

where $\vec{\xi}_{\infty}$ is a possibly branched smooth immersion of $\Sigma$. The maps $\vec{\eta}_{s}$ and $\vec{\zeta}_{t}$ are smooth, possibly branched, immersions of $S^{2}$; and $\theta_{t}$ is the integer density of the current $\left(\vec{\zeta}_{t}\right)_{*}\left[S^{2}\right]$ at some point $p_{t} \in$ $\vec{\zeta}_{t}\left(S^{2}\right)$, namely

$$
\theta_{t}:=\lim _{r \rightarrow 0} \frac{\mathcal{H}^{2}\left(B_{r}^{m}\left(p_{t}\right) \cap \vec{\zeta}_{t}\left(S^{2}\right)\right)}{\pi r^{2}} .
$$

The second part of our main result describes how the maps $\vec{\xi}_{\infty}, \vec{\eta}^{s}$ and $\vec{\zeta}^{t}$ are obtained from the original sequence $\vec{\Phi}_{k}$.

Theorem I.3 With the same notation as in theorem I.2, the immersion $\vec{\xi}_{\infty}$ is obtained as follows. There exist a sequence $f_{k}$ of diffeomorphisms of $\Sigma$, a sequence $\vec{\Xi}_{k}$ of Möebius transformations of $\mathbb{R}^{m}$, and finitely many points $\left\{a^{1}, \ldots, a^{n}\right\}$ such that $\Xi_{k} \circ \vec{\xi}_{k} \circ f_{k}$ is conformal and

$$
\vec{\xi}_{k}:=\Xi_{k} \circ \vec{\xi}_{k} \circ f_{k} \longrightarrow \vec{\xi}_{\infty} \quad \text { in } C_{l o c}^{l}\left(\Sigma \backslash\left\{a^{1}, \ldots, a^{n}\right\}\right) \quad \forall l \in \mathbb{N}
$$

Furthermore, there holds

$$
\lim _{k \rightarrow+\infty} W\left(\vec{\Phi}_{k}\right)=W\left(\vec{\xi}_{\infty}\right) \quad \Longleftrightarrow \quad \vec{\xi}_{k} \longrightarrow \vec{\xi}_{\infty} \quad \text { in } C^{l}(\Sigma) \quad \forall l \in \mathbb{N}
$$


Finally there exists a sequence $h_{k}$ of constant scalar curvature metric conformally equivalent to $\vec{\xi}_{k}=$ $\Xi_{k} \circ \vec{\xi}_{k} \circ f_{k}$ and strongly converging in $C^{l}(\Sigma)$, such that for any $s \in\{1, \ldots, p\}$ (resp. for any $t \in\{1, \ldots, q\}$ ) there exist a sequence of points $x_{k}^{s} \in \Sigma$ converging to one of the $a^{i}$, a sequence of radius $\rho_{k}^{s}$ converging to zero, and a sequence of Möbius transformations $\Xi_{k}^{s}$ (resp. $\Xi_{k}^{t}$ ) for which (in converging $h_{k}$ conformal coordinates $\varphi_{k}$ around $a^{i}$ ), one has

$$
\Xi_{k}^{s} \circ \vec{\xi}_{k} \circ \varphi_{k}\left(\rho_{k}^{s} y+\phi_{k}^{-1}\left(x_{k}^{s}\right)\right) \longrightarrow \vec{\eta}_{s} \circ \pi^{-1}(y) \quad \text { in } \quad C_{l o c}^{l}\left(\mathbb{C} \backslash\left\{a_{s}^{1}, \ldots, a_{s}^{n_{s}}\right\}\right)
$$

and respectively

$$
\Xi_{k}^{t} \circ \vec{\xi}_{k} \circ \varphi_{k}\left(\rho_{k}^{t} y+\phi_{k}^{-1}\left(x_{k}^{t}\right)\right) \longrightarrow \vec{\zeta}_{t} \circ \pi^{-1}(y) \quad \text { in } \quad C_{l o c}^{l}\left(\mathbb{C} \backslash\left\{a_{t}^{1}, \ldots, a_{t}^{n_{t}}\right\}\right)
$$

for any $l \in \mathbb{N} ;$ where $\pi$ denotes the stereographical projection from $S^{2}$ into $\mathbb{C}$ and $\left\{a_{s}^{1}, \ldots, a_{s}^{n_{s}}\right\}$ and $\left\{a_{t}^{1}, \ldots, a_{t}^{n_{t}}\right\}$ are finite sets of points in the complex plane.

The maps $\Xi_{k}^{s}$ are compositions of a dilation and isometries, while each map $\Xi_{k}^{t}$ is a composition of one inversion, one dilation, and one isometry.

The results given in theorem $[.2$ and theorem $[.3$ are to be viewed in the context of other bubble-neck decomposition and energy quantization situations previously studied. In particular for harmonic maps and other conformally invariant problems as those considered in $[\mathrm{SaU},[\mathrm{Jo},[\mathrm{St},[\mathrm{DiT},[\mathrm{Pa}, \mathrm{Ri5}$, and in Ri6. However, these problems are all of second-order elliptic or parabolic types. The novelty of the present work is an energy quantization result and bubble-neck decomposition for a fourth-order problem 4 . Moreover, the analysis which we present here requires to handle one additional delicate difficulty, namely the need to first control the conformal parameter in "conformally degenerating" neck regions (annuli), prior to obtaining from PDE techniques the vanishing of the energy in these regions.

The method we develop to prove theorem $I .2$ and theorem $[.3$ is partly inspired by a technique introduced by the second author in collaboration with F.H. Lin, in the context of the Ginzburg-Landau equations LiRi1 and in the context of harmonic maps LiRi2. This techniques relies on the properties of the Lorentz interpolation spaces. More precisely, in the present paper, we obtain estimates of the trace of the second fundamental form in the neck regions, respectively in the weak Marcinkiewicz space $L^{2, \infty}$ space, and in its predual, the Lorentz space $L^{2,1}$. These estimates constitute the main achievement of our proof. Prior to deriving these estimates, it is first necessary to carefully study the conformal factor of the conformal Willmore immersions in the neck region. This is done through extracting a good Coulomb gauge. The existence of such a gauge is one of the novelties of the present work, for it requires only the gradient of the Gauss map lie in $L^{2}$, and that it have a small $L^{2, \infty}$-norm. Such a control on the $L^{2, \infty}$-norm of the second fundamental form (i.e. of the gradient of the Gauss map) ultimately stems from the $e p$-regularity established in [Ri2]. The appropriate control in $L^{2,1}$ is then deduced using the standard methods of integrability by compensation and applying them to the conservation laws satisfied by Willmore immersions, and originally derived in $\mathrm{Ri} 2$.

It is legitimate to ask what happens if one removes the assumption on the control of the conformal structure. In this situation, the first important observation is provided by the examples constructed in $\mathrm{BuPa}$, where inverted catenoids arise as limiting bubbles, which are thus not smooth Willmore immersion of $S^{2}$. The second important observation is that harmonic mapping from a degenerating Riemann surface into a manifold are known in general not to satisfy the energy quantization property in the thin collar regions (cf. example constructed in $[\mathrm{Pa}$, and the careful systematic study given in [Zhu] ). Questions

${ }^{4}$ Indeed, in a conformal parametrization $\vec{\xi}$, the Willmore functional may be recast as

$$
W(\vec{\xi})=\frac{1}{4} \int_{\Sigma}\left|\Delta_{g} \vec{\xi}\right|^{2} d v o l_{g}
$$

thereby giving rise to a fourth-order problem 
associated with the more complex situation in which the sequence of conformal classes no longer remains within a subdomain of the Moduli space are considered in a forthcoming work [BR3].

To prevent the conformal class from degenerating, a sufficient energy condition has been given (in the cases $m=3$ and $m=4$ ) in KS1, and for surfaces in any codimension in Ri4 (and independently in [KuLi]). Following the convention in [Si], we introduce

$$
\beta_{g}^{m}:=\inf \{W(\vec{\Phi}) \quad ; \quad \vec{\Phi} \text { is an immersion of the genus } g \text { closed surface }\} .
$$

and

$$
\omega_{g}^{m}:=\min \left\{4 \pi+\sum_{i=1}^{p}\left(\beta_{g_{i}}^{m}-4 \pi\right) \quad ; \quad g=g_{1}+\ldots+g_{p} \quad, \quad 1 \leq g_{i}<g\right\} .
$$

In [BK], it is proved that for $g \geq 2$ there holds

$$
\beta_{g}^{m}<\omega_{g}^{m}
$$

Theorem I.4 Ri4] [KuLi] Let $\left(\Sigma, c_{k}\right)$ be a sequence of closed Riemann surfaces of genus $g$, with degenerating conformal class $\left[c_{k}\right]$ diverging to the boundary of the Moduli space of $\Sigma$. Let $\vec{\Phi}_{k}$ be a sequence of conformal immersions from $\left(\Sigma, c_{k}\right)$ into $\mathbb{R}^{m}$. Then

$$
\liminf _{k \rightarrow+\infty} \int_{\Sigma}\left|\vec{H}_{\vec{\Phi}_{k}}\right|^{2} d v o l_{\vec{\Phi}_{k}^{*} g_{\mathbb{R}} m} \geq \min \left\{8 \pi, \omega_{g}^{m}\right\}
$$

A consequence of our analysis below is the following result.

Theorem I.5 Let $\Sigma$ be an arbitrary closed two-dimensional manifold. Modulo the action of the Möbius group of $\mathbb{R}^{m}$, the space of Willmore immersions into $\mathbb{R}^{m}$, for $m=3$ and $m=4$, satisfying

$$
W(\Phi)<\min \left\{8 \pi, \omega_{g}^{m}\right\}-\delta
$$

is strongly compact in the $C^{l}$ topology for any $l \in \mathbb{N}$ and any $\delta>0$.

This result was obtained for $\Sigma=T^{2}$ and $m=3$ in [KS] and for $\Sigma=T^{2}$ and $m=4$ in [Ri2].

The paper is organized as follows. In section II, we recall the formalism introduce in Ri3 which allow us to appropriately "renormalize" a sequence of weak immersions uniformly bounded Willmore energy. In section III, we outline a generic "energy tracking" procedure enabling us to detect bubbles and neck regions. Section IV, also general in nature, explains how to construct a Coulomb moving frame under the hypothesis that the Gauss map has small weak- $L^{2}$-norm. In Section V, we develop uniform Harnack estimates giving control of the conformal factor of weak immersions in the neck regions. Willmore immersions first appear in section VI, where we show how to control the $L^{2,1}$-norm of the mean curvature vector in the neck regions. In Section VII, we derive an energy quantization result for a sequence of Gauss maps corresponding to a sequence of Willmore immersions. Finally, in section VIII, all of the aforementioned estimates are seamed together, and the main theorems $[.2$ and $[.3$ are proved.

Acknowledgements. This work was partly elaborated while the first author was visiting the Forschungsinstitut für Mathematik at the ETH Zürich. He would like to thank the institute for it's hospitality and the excellent working conditions. 


\section{Preliminaries}

In $[\mathrm{Ri3}$, the second author developed a suitable framework in which one can perform the calculus of variation of the Willmore Lagrangian of a surface. In this framework, a particularly useful space is that of Lipschitz immersions with $L^{2}$-bounded second fundamental forms.

Let $g_{0}$ be a smooth "reference" metric on $\Sigma$. One defines the Sobolev spaces $W^{k, p}\left(\Sigma, \mathbb{R}^{m}\right)$ of measurable maps from $\Sigma$ into $\mathbb{R}^{m}$ as

$$
W^{k, p}\left(\Sigma, \mathbb{R}^{m}\right):=\left\{f \text { meas. } \Sigma \rightarrow \mathbb{R}^{m} \text { s.t. } \sum_{l=0}^{k} \int_{\Sigma}\left|\nabla^{l} f\right|_{g_{0}}^{p} d v o l_{g_{0}}<+\infty\right\} .
$$

As $\Sigma$ is assumed to be compact, this space is independent of the chosen reference metric $g_{0}$.

It is important to have a weak first fundamental form: we need $\vec{\Phi}^{*} g_{\mathbb{R}^{m}}$ to define an $L^{\infty}$ metric with a bounded inverse. This is the case if we assume that $\vec{\Phi}$ lies in $W^{1, \infty}(\Sigma)$ and if $d \vec{\Phi}$ has maximal rank 2 at every point, with some uniform quantitative control of "how far" $d \vec{\Phi}$ is from being degenerate. Namely, there exists $c_{0}>0$ such that

$$
|d \vec{\Phi} \wedge d \vec{\Phi}|_{g_{0}} \geq c_{0}>0
$$

where $d \vec{\Phi} \wedge d \vec{\Phi}$ is a 2 -form on $\Sigma$ with values in the space of 2 -vectors of $\mathbb{R}^{m}$, and given in local coordinates by $2 \partial_{x} \vec{\Phi} \wedge \partial_{y} \vec{\Phi} d x \wedge d y$. Note that the condition (II.1) is independent of the choice of the metric $g_{0}$. To a Lipschitz immersion satisfying (II.1) we associate its Gauss map

$$
\vec{n}_{\vec{\Phi}}:=\star \frac{\partial_{x_{1}} \vec{\Phi} \wedge \partial_{x_{2}} \vec{\Phi}}{\left|\partial_{x_{1}} \vec{\Phi} \wedge \partial_{x_{2}} \vec{\Phi}\right|}
$$

It belongs to $L^{\infty}(\Sigma)$ and takes values in the Grassmannian of oriented $(m-2)$-planes in $\mathbb{R}^{m}$.

We next introduce the space $\mathcal{E}_{\Sigma}$ of Lipschitz immersions 5 of $\Sigma$ with square-integrable second fundamental form:

$$
\mathcal{E}_{\Sigma}:=\left\{\begin{array}{cc}
\vec{\Phi} \in W^{1, \infty}\left(\Sigma, \mathbb{R}^{m}\right) & \text { s.t. } \vec{\Phi} \text { satisfies (II.1) for some } c_{0} \\
\text { and } \int_{\Sigma}|d \vec{n}|_{g}^{2} d v o l_{g}<+\infty
\end{array}\right\} .
$$

As before, $g:=\vec{\Phi}^{*} g_{\mathbb{R}^{m}}$ is the pull-back by $\vec{\Phi}$ of the flat canonical metric $g_{\mathbb{R}^{m}}$ of $\mathbb{R}^{m}$, and $d v o l_{g}$ is its corresponding volume form.

As in the case of the Yang-Mills functional, one of the main difficulties one encounters in the variational study of the Willmore functional is the size of the corresponding gauge group (i.e. the group of transformations through which the energy is invariant). For the energy $W$, this gauge group is very large: it contains all diffeomorphisms of $\Sigma$. To "break" the gauge invariance, one of the first techniques considered in Ri3. consists in working with conformal immersions. Indeed, owing to the work of Müller and Sverak [MS] and to the work of Hélein [Hel], one gains an $L^{\infty}$-control of the conformal parameter under a small energy $E$ assumption. It is then possible to show that the pull-back metric of any element in $\mathcal{E}_{\Sigma}$ defines a smooth conformal structure on $\Sigma$ (cf. details in Ri1]). Thus, corresponding to any element $\vec{\Phi} \in \mathcal{E}_{\Sigma}$, there exists a Lipschitz diffeomorphism $f$ of $\Sigma$ such that $\vec{\Phi} \circ f$ is conformal. Having found a conformal structure $c$ of $\Sigma$, one defines

$$
\mathcal{E}_{\Sigma}^{c}:=\left\{\vec{\Phi} \in \mathcal{E}_{\Sigma} \quad \text { s.t. } \vec{\Phi} \text { is conformal w.r.t. } c\right\} .
$$

\footnotetext{
${ }^{5}$ henceforth called weak immersions.
} 
Naturally, in considering a sequence of elements in $\mathcal{E}_{\Sigma}$, the corresponding sequence of conformal classes it yields is not a priori controlled, and it might diverge in the moduli space of $\Sigma$, even when the Willmore energy of the immersions is uniformly bounded. This possible degeneracy of the conformal class is another source of trouble when trying to control a sequence of immersions with uniformly bounded Willmore energy. In the present work, we will assume that the aforementioned sequence of conformal classes does not degenerate. Sufficient conditions to avoid degeneracy of the conformal structure were given in [Ri4], and independently in $\mathrm{KuLi}$.

Once the global gauge choice is made, the second difficulty one encounters is the invariance of the Willmore functional under conformal transformations of $\mathbb{R}^{m}$. Namely, any conformal diffeomorphism $\Xi$ of $\mathbb{R}^{m}$ (i.e. an element of the Möbius group of $\mathbb{R}^{m}$ ) leaves both $\mathcal{E}_{\Sigma}^{c}$ and $W$ (through composition). Since the Möbius group is non-compact, this invariance generates serious troubles in the study of a sequence of immersions with uniformly bounded Willmore energy. Indeed, such a sequence may a-priori collapse to a point in the limit! To overcome this difficulty, a method for extracting a suitable $\Xi$ was devised in Lemma A.4 of [Ri3]. Combined with the result of Lemma III.1 in [Ri3], one obtains an "almost" weak closure result for sequences in $\mathcal{E}_{\Sigma}^{c}$ modulo composition with suitable elements of the Möbius group. More precisely, the limit immersion is not necessarily a weak immersion of the whole $\Sigma$, but only a weak immersion away from possibly finitely many points. We are thus led to consider the space of weak branched immersions,

$$
\mathcal{F}_{\Sigma}:=\left\{\begin{array}{c}
\vec{\Phi} \in W^{1, \infty}\left(\Sigma, \mathbb{R}^{m}\right) \quad \text { s.t. } \exists\left\{a_{1}, \ldots, a_{N}\right\} \in \Sigma \text { with } \\
\forall K \text { compact subset of } \Sigma \backslash\left\{a_{1}, \ldots, a_{N}\right\} \\
\exists c_{K}>0 \quad \text { s.t. } \forall x \in K \quad|d \vec{\Phi} \wedge d \vec{\Phi}|_{g_{0}}(x) \geq c_{K} \\
\text { and } \int_{\Sigma}|d \vec{n}|_{g}^{2} d v o l_{g}<+\infty
\end{array}\right\} .
$$

Finally, we introduce the space of elements in $\mathcal{F}_{\Sigma}$ which are conformal with respect to some fixed conformal structure $c$,

$$
\mathcal{F}_{\Sigma}^{c}:=\left\{\vec{\Phi} \in \mathcal{F}_{\Sigma} \quad \text { s. t. } \quad \vec{\Phi} \text { is conformal w.r.t. } c\right\} .
$$

The above discussion is summarized in the following result, which will be a central ingredient in the rest of this article.

Lemma II.1 Ri3] Let $\Sigma$ be a closed two-dimensional manifold. Let $\vec{\Phi}_{k}$ be a sequence of elements in $\mathcal{E}_{\Sigma}$ such that $W\left(\Phi_{k}\right)$ is uniformly bounded. Assume that the conformal class of the conformal structure $c_{k}$ (i.e. complex structure of $\Sigma$ ) defined by $\vec{\Phi}_{k}$ remains in a compact subspace of the Moduli space of $\Sigma$. Then, modulo extraction of a subsequence, the sequence $c_{k}$ converges to a smooth limiting complex structure $c_{\infty}$; and there exist a sequence of Lipschitz diffeomorphisms $f_{k}$ of $\Sigma$ and a sequence of smooth conformal structures $c_{k}$ of $\Sigma$ such that $\vec{\Phi}_{k} \circ f_{k}$ is conformal from $\left(\Sigma, c_{k}\right)$ into $\mathbb{R}^{m}$. Moreover, there exists a sequence $\Xi_{k}$ of conformal diffeomorphisms of $\mathbb{R}^{m} \cup\{\infty\}$ and at most finitely many points $\left\{a^{1}, \ldots, a^{n}\right\}$ such that

$$
\limsup _{k \rightarrow+\infty} \mathcal{H}\left(\Xi_{k} \circ \vec{\Phi}_{k} \circ f_{k}(\Sigma)\right)<+\infty \quad, \quad \Xi_{k} \circ \vec{\Phi}_{k} \circ f_{k}(\Sigma) \subset B_{R}(0)
$$

for some $R>0$ independent of $k$, and

$$
\vec{\xi}_{k}:=\Xi_{k} \circ \vec{\Phi}_{k} \circ f_{k} \rightarrow \vec{\xi}_{\infty} \quad \text { weakly in }\left(W_{l o c}^{2,2} \cap W_{l o c}^{1, \infty}\right)^{*}\left(\Sigma \backslash\left\{a^{1}, \ldots, a^{n}\right\}\right) .
$$


The convergences are understood with respect to $h_{k}$, which is the constant scalar curvature metric of unit volume attached to the conformal structure $c_{k}$.

Furthermore, there holds

$$
\forall K \text { compact subset of } \Sigma \backslash\left\{a^{1}, \ldots, a^{n}\right\} \quad \limsup _{k \rightarrow+\infty}\left\|\log \left|d \vec{\xi}_{k}\right|_{h_{k}}\right\|_{L^{\infty}(K)}<+\infty \quad .
$$

Finally, $\vec{\xi}_{\infty}$ is a weak immersion of $\Sigma \backslash\left\{a^{1}, \ldots, a^{n}\right\}$ and conformal from $\left(\Sigma, c_{\infty}\right)$ into $\mathbb{R}^{m}$.

\section{The bubble-neck decomposition procedure}

The goal of this section is to establish the following technical proposition. Although its statement might seem at first somewhat overwhelming, its proof involves no particularly challenging difficulties and is based on an iteration argument.

Proposition III.1 [Bubble-neck decomposition] Let $\Sigma$ be a closed two-dimensional manifold. Let $\vec{\xi}_{k}$ be a sequence of weak Lipschitz immersions with $L^{2}$-bounded second fundamental forms (i.e. $\vec{\xi}_{k} \in \mathcal{E}_{\Sigma}$ ) such that

$$
\int_{\Sigma}\left|d \vec{n}_{\vec{\xi}_{k}}\right|_{g_{k}}^{2} d v o l_{g_{k}} \leq \Lambda
$$

where $g_{k}:=\vec{\xi}_{k}^{*} g_{\mathbb{R}^{m}}$. We suppose that $g_{k}$ is conformally equivalent to a constant scalar curvature metric $h_{k}$ such that for all $l \in \mathbb{N}$

$$
h_{k} \longrightarrow h_{\infty} \quad \text { in } C^{l}(\Sigma),
$$

where $h_{\infty}$ is a constant scalar curvature on $\Sigma$.

Suppose there exist $n$ points $\left\{a^{1}, \ldots, a^{n}\right\} \subset \Sigma$ and a radius $R>0$ such that the following holds

$$
\limsup _{k \rightarrow+\infty} \mathcal{H}\left(\vec{\xi}_{k}(\Sigma)\right)<+\infty
$$

(ii)

$$
\vec{\xi}_{k}(\Sigma) \subset B_{R}(0)
$$

(iii)

$$
\vec{\xi}_{k} \rightarrow \vec{\xi}_{\infty} \quad \text { weakly in }\left(W_{l o c}^{2,2} \cap W_{l o c}^{1, \infty}\right)^{*}\left(\Sigma \backslash\left\{a^{1},, \ldots, a^{n}\right\}\right)
$$

(iv)

$$
\forall K \text { compact subset of } \Sigma \backslash\left\{a^{1}, \ldots, a^{n}\right\} \quad \limsup _{k \rightarrow+\infty}\left\|\log \left|d \vec{\xi}_{k}\right|_{h_{k}}\right\|_{L^{\infty}(K)}<+\infty \quad
$$

Then for any $0<\varepsilon_{0}<8 \pi / 3$, there exist a subsequence, still denoted $\vec{\xi}_{k}, n$ integers $\left\{Q^{1}, \ldots, Q^{n}\right\}, n$ sequences of points $\left(x_{k}^{i, j}\right)_{j=1, \ldots, Q^{i}} \subset \Sigma$, and $n$ sequences of radii $\left(\rho_{k}^{i, j}\right)_{j=1, \ldots, Q^{i}}$ satisfying

$$
\begin{array}{ll}
\forall i \in 1, \ldots, n \quad \forall j \in\left\{1, \ldots, Q^{i}\right\} & \lim _{k \rightarrow+\infty} x_{k}^{i, j}=a^{i}, \\
\forall i \in 1, \ldots, n \quad \forall j \in\left\{1, \ldots, Q^{i}\right\} & \lim _{k \rightarrow+\infty} \rho_{k}^{i, j}=0,
\end{array}
$$


and

$$
\forall i \in 1, \ldots, n \quad \forall j \neq j^{\prime} \in\left\{1, \ldots, Q^{i}\right\} \quad\left\{\begin{array}{c}
\text { either } \lim _{k \rightarrow+\infty} \frac{\rho_{k}^{i, j}}{\rho_{k}^{i, j^{\prime}}}+\frac{\rho_{k}^{i, j^{\prime}}}{\rho_{k}^{i, j}}=+\infty \\
\text { or } \lim _{k \rightarrow+\infty} \frac{\left|x_{k}^{i, j}-x_{k}^{i, j^{\prime}}\right|}{\rho_{k}^{i, j}+\rho_{k}^{i, j^{\prime}}}=+\infty
\end{array}\right.
$$

Moreover,

$$
\forall i \in\{1, \ldots, n\} \quad \forall j \in\left\{1, \ldots, Q^{i}\right\} \quad \int_{B_{\rho_{k}, j}\left(x_{k}^{i, j}\right)}\left|d \vec{n}_{\vec{\xi}_{k}}\right|_{g_{k}}^{2} d v o l_{g_{k}}>\varepsilon_{0}
$$

The set of balls $B_{\rho_{k}^{i, j}}$ are called "bubbles" associated to the sequence $\vec{\xi}_{k}$.

We have furthermore that for any $i \in\{1, \ldots, n\}$ and for any $j \in\left\{1, \ldots, Q^{i}\right\}$, the set of indice 6

$$
I^{i, j}:=\left\{j^{\prime} \quad ; \quad x_{k}^{i, j^{\prime}} \in B_{\rho_{k}^{i, j}}\left(x_{k}^{i, j}\right) \quad ; \quad \frac{\rho_{k}^{i, j}}{\rho_{k}^{i, j^{\prime}}} \rightarrow+\infty\right\}
$$

is independent of $k$. It is called the set of "bubbles contained in the bubble $B_{\rho_{k}^{i, j}}\left(x^{i, j}\right)$ ".

For any $\alpha<1$ we denote

$$
\forall i \in\{1, \ldots, n\} \quad \Omega_{k}^{i}(\alpha):=B_{\alpha}\left(a^{i}\right) \backslash \bigcup_{j=1}^{Q^{i}} B_{\alpha^{-1}} \rho_{k}^{i, j}\left(x_{k}^{i, j}\right)
$$

and

$$
\forall i \in\{1, \ldots, n\} \quad \forall j \in\left\{1, \ldots, Q^{i}\right\} \quad \Omega_{k}^{i, j}(\alpha):=\bigcup_{j^{\prime} \in I^{i, j}}\left(B_{\alpha \rho_{k}^{i, j}}\left(x_{k}^{i, j^{\prime}}\right) \backslash \bigcup_{j^{\prime \prime} \in I^{i, j}} B_{\alpha^{-1} \rho_{k}^{i, j^{\prime \prime}}}\left(x_{k}^{i, j^{\prime \prime}}\right)\right) .
$$

The sets $\Omega_{k}^{i}(\alpha)$ and the $\Omega_{k}^{i, j}(\alpha)$ are the " $\alpha$-neck regions" of $\vec{\xi}_{k}$. Let

$$
\Omega_{k}(\alpha):=\bigcup_{i=1}^{n}\left(\Omega_{k}^{i}(\alpha) \cup \bigcup_{j=1}^{Q^{i}} \Omega_{k}^{i, j}(\alpha)\right)
$$

Then there exists $0<\alpha<1$ independent of $k$, such that

$$
\begin{aligned}
& \forall i \in\{1, \ldots, n\} \quad \forall j \in\left\{1, \ldots, Q^{i}\right\} \quad \text { and } \forall \rho>0 \quad \text { with } \quad B_{2 \rho}\left(x_{k}^{i, j}\right) \backslash B_{\rho}(x) \subset \Omega_{k}(\alpha) \\
& \text { then } \quad \int_{B_{2 \rho}\left(x_{k}^{i, j}\right) \backslash B_{\rho}(x)}\left|d \vec{n}_{\vec{\xi}_{k}}\right|_{g_{k}}^{2} d v o l_{g_{k}}<\varepsilon_{0} \quad,
\end{aligned}
$$

and such that

$$
\begin{aligned}
& \forall i \in\{1, \ldots, n\} \quad \forall j \in\left\{1, \ldots, Q^{i}\right\} \\
& \text { then } \quad \int_{B_{\rho_{k}, j}^{i, j}\left(x_{k}^{i, j}\right) \backslash \bigcup_{j^{\prime} \in I^{i, j}} B_{\alpha \rho_{k} i, j}\left(x_{k}^{i, j^{\prime}}\right)}\left|d \vec{n}_{\vec{\xi}_{k}}\right|_{g_{k}}^{2} d v o l_{g_{k}}>\varepsilon_{0} .
\end{aligned}
$$

\footnotetext{
${ }^{6}$ this set might be empty.
} 
Given $0<\alpha<1$, we set

$$
\begin{aligned}
& \forall i \in\{1, \ldots, n\} \quad \forall j \in\left\{1, \ldots, Q^{i}\right\} \\
& r_{k}^{i, j}:=\inf \left\{\begin{array}{c}
B_{\rho}(x) \subset B_{\alpha^{-1} \rho_{k}^{i, j}}\left(x_{k}^{i, j}\right) \backslash \bigcup_{j^{\prime} \in I^{i, j}} B_{\alpha \rho_{k}^{i, j}}\left(x_{k}^{i, j^{\prime}}\right) \\
0<r<\rho_{k}^{i, j} ; \quad \int_{B_{r}(x)}\left|d \vec{n}_{\vec{\xi}_{k}}\right|_{g_{k}}^{2} d v o l_{g_{k}}=\varepsilon_{0}
\end{array}\right\} .
\end{aligned}
$$

Then there holds

$$
\forall i \in\{1, \ldots, n\} \quad \forall j \in\left\{1, \ldots, Q^{i}\right\} \quad \liminf _{k \rightarrow+\infty} \frac{r_{k}^{i, j}}{\rho_{k}^{i, j}}>0
$$

We prove this proposition inductively by repeating finitely many iterations of the following lemma.

Lemma III.2 [Energy tracking procedure] Let $\vec{\xi}_{k}$ be a sequence conformal weak immersions from the two-dimensional annulus $\Omega_{k}:=B_{R_{k}}(0) \backslash B_{r_{k}}(0) \subset \mathbb{C}$ into $\mathbb{R}^{m}$, with $R_{k} / r_{k} \rightarrow+\infty$. We assume that

$$
\int_{B_{R_{k}}(0) \backslash B_{r_{k}}(0)}\left|\nabla \vec{n}_{\vec{\xi}_{k}}\right|^{2} d x \leq \Lambda<+\infty \text {. }
$$

Then for any $\varepsilon_{0}>0$, one can extract a subsequence, still denoted $\vec{\xi}_{k}$, such that there exist a fixed integer $N \in \mathbb{N}$ satisfying

$$
N \leq 4\left(\frac{\Lambda}{\varepsilon_{0}}+1\right),
$$

and a sequence of decreasing families of $(N+1)$ radii

$$
R_{k}^{0}=R_{k}>R_{k}^{1}>\ldots>R_{k}^{N}=r_{k}
$$

for which

$$
\forall i \in\{0, \ldots, N-1\} \quad \int_{\partial B_{R_{k}^{i}}}\left|\nabla \vec{n}_{\vec{\xi}_{k}}\right| d \mathcal{H}^{1}<\sqrt{\frac{5 \pi}{2} \varepsilon_{0}} .
$$

Moreover, the set $I=\{0, \ldots, N-1\}$ can be decomposed into two disjoint subsets $I:=I_{0} \cup I_{1}$ with

$$
\forall i \in I_{0} \quad \log _{2} \frac{4}{3}<\tau_{i}:=\lim _{k \rightarrow+\infty} \log \frac{R_{k}^{i}}{R_{k}^{i+1}}<+\infty \quad,
$$

and

$$
\forall i \in I_{1} \quad \int_{B_{R_{k}^{i}} \backslash B_{R_{k}^{i+1}}}\left|\nabla \vec{n}_{\vec{\xi}_{k}}\right|^{2} d x<\varepsilon \quad \text { and } \quad \lim _{k \rightarrow+\infty} \log \frac{R_{k}^{i}}{R_{k}^{i+1}}=+\infty
$$

Proof of lemma III.2, We begin by constructing a decreasing sequence of radii $\left\{\rho_{k}^{0}=R_{k}>\rho_{k}^{1}>\ldots>\right.$ $\left.\rho_{k}^{Q-1}>\rho_{k}^{Q}=r_{k}\right\}$ such that

$$
\forall j \in\{0, \ldots, Q\} \quad \int_{\partial B_{\rho_{k}^{j}}(0)}\left|\nabla \vec{n}_{\vec{\xi}_{k}}\right| d \mathcal{H}^{1}<\sqrt{\frac{5 \pi}{2} \varepsilon_{0}}
$$


and

$$
\forall j \in\{0, \ldots, Q\} \quad \log _{2} \frac{\rho_{k}^{j-1}}{\rho_{k}^{j}}>\log _{2} \frac{4}{3} .
$$

Moreover, for any $j \in J=\{0, \ldots, Q-1\}$, one of the following three possibilities holds

(i)

$$
\varepsilon_{0} / 2<\int_{B_{\rho_{k}^{j}} \backslash B_{\rho_{k}^{j+1}}}\left|\nabla \vec{n}_{\vec{\xi}_{k}}\right|^{2} d x<\varepsilon_{0}
$$

(ii)

$$
\int_{B_{\rho_{k}^{j}} \backslash B_{\rho_{k}^{j+1}}}\left|\nabla \vec{n}_{\vec{\xi}_{k}}\right|^{2} d x<\varepsilon_{0} / 2
$$

and either $\rho_{k}^{j+1}=\rho_{k}^{Q}=r_{k}$ or

$$
\int_{B_{\rho_{k}^{j+1}} \backslash B_{\rho_{k}^{j+2}}}\left|\nabla \vec{n}_{\vec{\xi}_{k}}\right|^{2} d x>\varepsilon_{0} / 2 \quad \text { and } \quad \log _{2} \frac{\rho_{k}^{j+1}}{\rho_{k}^{j+2}} \leq C
$$

(iii)

$$
\int_{B_{\rho_{k}^{j}} \backslash B_{\rho_{k}^{j+1}}}\left|\nabla \vec{n}_{\vec{\xi}_{k}}\right|^{2} d x>\varepsilon_{0} / 2 \quad \text { and } \quad \log _{2} \frac{\rho_{k}^{j}}{\rho_{k}^{j+1}} \leq C
$$

where 7 $C:=\left[2 \Lambda / \varepsilon_{0}\right]+2$.

The construction of $\rho_{k}^{j}$ is done by induction on $j$. We take first $\rho_{k}^{0}:=R_{k}$. Assume that we have found some $\rho_{k}^{j}$ satisfying (III.21)-(III.22) and one of the alternatives (i)-(iii) for $l \leq j$, and such that (III.24) fails for $j$. To construct $\rho_{k}^{j+1}$, we proceed as follows. Let $\rho \geq r_{k}$ be such that

$$
\int_{B_{\rho_{k}^{j}} \backslash B_{\rho}(0)}\left|\nabla \vec{n}_{\vec{\xi}_{k}}\right|^{2} d x=\varepsilon_{0} / 2
$$

If no such $\rho$ exists, we simply choose $\rho_{k}^{j+1}=r_{k}$.

For any $0<\alpha<1$ and $R_{k}>t>\alpha^{-1} r_{k}$, there holds

$$
\int_{\alpha t}^{t} d s \int_{\partial B_{s}(0)}\left|\nabla \vec{n}_{\vec{\xi}_{k}}\right| \leq t \sqrt{\pi\left(1-\alpha^{2}\right)} \sqrt{\int_{B_{t} \backslash B_{\alpha t}}\left|\nabla \vec{n}_{\vec{\xi}_{k}}\right|^{2}} .
$$

Suppose first that

$$
\int_{B_{\rho} \backslash B_{\rho / 2}}\left|\nabla \vec{n}_{\vec{\xi}_{k}}\right|^{2} \leq \varepsilon_{0} / 2
$$

Applying (III.27) with $t=3 \rho / 4$ and $\alpha=2 / 3$, and using the mean value theorem, yields some $s \in$ $[\rho / 2,3 \rho / 4]$ such that

$$
\int_{\partial B_{s}(0)}\left|\nabla \vec{n}_{\vec{\xi}_{k}}\right| d \mathcal{H}^{1}<\sqrt{\frac{5 \pi}{2} \varepsilon_{0}} .
$$

Then $\rho_{k}^{j+1}:=s$ satisfies (III.21), (III.22) and the alternative (III.23).

\footnotetext{
${ }^{7}[a]$ denotes the integer part of $a \in \mathbb{R}$, namely, the largest integer less than or equal to $a$.
} 
Suppose next that

$$
\int_{B_{\rho} \backslash B_{\rho / 2}}\left|\nabla \vec{n}_{\vec{\xi}_{k}}\right|^{2}>\varepsilon_{0} / 2
$$

Then there must exist some $l \in\left\{1, \ldots,\left[2 \Lambda / \varepsilon_{0}\right]+1\right\}$ for which

$$
\int_{B_{2-l} \backslash B_{2-l-1} \rho}\left|\nabla \vec{n}_{\vec{\xi}_{k}}\right|^{2} \leq \varepsilon_{0} / 2
$$

Calling again upon (III.27) with $t=2^{-l} \rho$ and $\alpha=1 / 2$, and using the mean value theorem, we obtain some $s \in\left[2^{-l-1}, 2^{-l}\right]$ such that

$$
\int_{\partial B_{s}(0)}\left|\nabla \vec{n}_{\vec{\xi}_{k}}\right| d \mathcal{H}^{1}<\sqrt{\frac{3 \pi}{2} \varepsilon_{0}} .
$$

If $\rho_{k}^{j} \leq 2 \rho$, then we let $\rho_{k}^{j+1}:=s$, and note that (III.21), (III.22), and the third alternative (III.26) with $C=\left[2 \Lambda / \varepsilon_{0}\right]+2$ are satisfied. If instead $\rho_{k}^{j}>2 \rho$, we pick some $\sigma \in[2 \rho, \rho]$ such that

$$
\int_{\partial B_{\sigma}(0)}\left|\nabla \vec{n}_{\vec{\xi}_{k}}\right| d \mathcal{H}^{1}<\sqrt{\frac{3 \pi}{2} \varepsilon_{0}}
$$

and set $\rho_{k}^{j+1}:=\sigma$ and $\rho_{k}^{j+2}:=s$. One verifies that (II.21), (III.22), and the second alternative (III.24)(III.25) is satisfied.

The above inductive construction must stop after a number of steps smaller than $\left[4 \Lambda / \varepsilon_{0}\right]+2$. Indeed, at least every other index, an amount of $\varepsilon_{0} / 2$ of the (finite) Dirichlet energy of $\vec{n}_{\vec{\xi}_{k}}$ is exhausted.

To complete the proof of the lemma, we first extract a subsequence $\vec{\xi}_{k^{\prime}}$ such that

- $Q$ is independent of $k^{\prime}$.

- for each $j \in\{0, \ldots, Q-1\}$, we have

$$
\tau_{j}:=\lim _{k^{\prime} \rightarrow+\infty} \log _{2} \frac{\rho_{k^{\prime}}^{j}}{\rho_{k^{\prime}}^{j+1}} \in \mathbb{R} \cup\{+\infty\} .
$$

We keep denoting this subsequence by $\vec{\xi}_{k}$. From the sequence $\rho_{k}^{j}$, we extract the desired decreasing sequence of radii $R_{k}^{j}$ by merging successive annuli such that

$$
\lim _{k^{\prime} \rightarrow+\infty} \log _{2} \frac{\rho_{k^{\prime}}^{j}}{\rho_{k^{\prime}}^{j+1}}=\tau_{j} \in \mathbb{R}
$$

and dropping the intermediate $\rho_{k}^{j}$. One easily verifies that the obtained sequence $R_{k}^{j}$ satisfies (III.17)(III.20), thereby completing the proof of lemma III.2.

\section{Proof of proposition III.1.}

Let $0<\varepsilon_{0}<8 \pi / 3$. Consider

$$
\rho_{k}:=\inf \left\{\rho ; \int_{B_{\rho}(x)}\left|d \vec{n}_{\vec{\xi}_{k}}\right|_{g_{k}}^{2} d v o l_{g_{k}}=\varepsilon_{0} \quad \forall x \in \Sigma\right\},
$$


where $B_{\rho}(x)$ denotes the geodesic ball of center $x$ and radius $\rho$ for the constant scalar curvature $h_{k}$. If

$$
\limsup _{k \rightarrow+\infty} \rho_{k}>0
$$

we can extract a subsequence such that $\rho_{k}$ converges to a positive constant. Then there is no $a_{i}$. This would indeed require the concentration of at least $8 \pi / 3$ of the energy, which is precluded from the result proved in [Ri3]. There is no bubble and the procedure stops.

Alternatively, we consider the case when

$$
\lim _{k \rightarrow+\infty} \rho_{k}=0
$$

Let $x_{k} \in \Sigma$ be such that

$$
\int_{B_{\rho_{k}}\left(x_{k}\right)}\left|d \vec{n}_{\vec{\xi}_{k}}\right|_{g_{k}}^{2} d v o l_{g_{k}}=\varepsilon_{0}
$$

Modulo the extraction of a subsequence, we have that $x_{k}$ converges to one of the points $a^{i}$. We set $x_{k}^{i, 1}:=x_{k}$ and $\rho_{k}^{i, 1}:=\rho_{k}$. By hypothesis, we may choose converging conformal coordinates around $a^{i}$. For notational convenience and clarity, since the converging metrics $h_{k}$ are uniformly equivalent to the flat metric, we shall work with flat geodesic balls. We can choose a subsequence $\vec{\xi}_{k}$ and a fixed radius $\alpha>0$ such that, for any

$$
\lim _{k \rightarrow+\infty} \sup _{0<r \leq \alpha}\left\{r ; \quad \int_{B_{\alpha}(0) \backslash B_{r}(0)}\left|\nabla \vec{n}_{\vec{\xi}_{k}}\right|^{2} d x=\varepsilon_{0}\right\}=0
$$

and we apply lemma III.2 to this subsequence, still denoted $\vec{\xi}_{k}$, and to $R_{k}:=\alpha$ and $r_{k}:=\rho_{k}^{i, 1}=\rho_{k}$. This gives then the existence of this family of radii

$$
R_{k}^{0}=\alpha>R_{k}^{1}>\ldots>R_{k}^{N}=\rho_{k}^{i, 0}
$$

satisfying (III.18)-(III.20) and

$$
\lim _{k \rightarrow+\infty} \log \frac{R_{k}^{0}}{R_{k}^{1}}=+\infty .
$$

We merge successive radii $R_{k}^{i}$ and $R_{k}^{i+1}$ for $i \in I_{1}$ or for $i \in I_{0}$ such that

$$
\int_{B_{R_{k}^{i}}\left(x_{k}^{i, 1}\right) \backslash B_{R_{k}^{i+1}}\left(x_{k}^{i, 1}\right)}\left|\nabla \vec{n}_{\vec{\xi}}\right|^{2} d x_{1} d x_{2} \leq \varepsilon_{0}
$$

We then get a subsequence of radii $R_{k}^{0}=\alpha>R_{k}^{i_{1}}>, \ldots,>R_{k}^{i_{P}}=\rho_{k}^{i, 0}$ for $P \leq N$ such that either, for $i_{l} \in I_{0}$

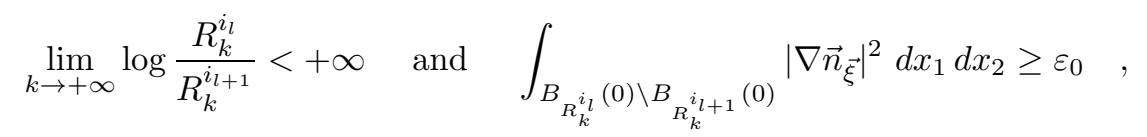

or, when $i_{l} \in I_{1}$, we have

$$
\lim _{k \rightarrow+\infty} \log \frac{R_{k}^{i_{l}}}{R_{k}^{i_{l+1}}}=+\infty \quad \text { and } \quad \forall \rho \in\left(R_{k}^{i_{l+1}}, R_{k}^{i_{l}} / 2\right) \quad \int_{B_{2 \rho}\left(x_{k}^{i, 1}\right) \backslash B_{\rho}\left(x_{k}^{i, 1}\right)}\left|\nabla \vec{n}_{\vec{\xi}}\right|^{2} d x_{1} d x_{2}<\varepsilon_{0}
$$

We consider the smallest annulus $\Omega_{k}^{i_{l}}:=B_{R_{k}^{i_{l}}} \backslash B_{R_{k}^{i_{l+1}}}$ of the first type $i_{l} \in I_{0}$. For such an $i_{l}$ we introduce

$$
\rho_{k}^{i_{l}}:=\inf \left\{\rho<R_{k}^{i_{l}+1} ; \int_{B_{\rho}(x)}\left|d \vec{n}_{\vec{\xi}_{k}}\right|_{g_{k}}^{2} d v o l_{g_{k}}=\varepsilon_{0} \quad \text { where } \quad x \in \Omega_{k}^{i_{l}}\right\}
$$


We now consider the following alternative. Either

$$
\limsup _{k \rightarrow+\infty} \frac{\rho_{k}^{i_{l}}}{R_{k}^{i_{l}}}>0
$$

we then extract a subsequence still denoted $\vec{\xi}_{k}$ such that

$$
\lim _{k \rightarrow+\infty} \frac{\rho_{k}^{i_{l}}}{R_{k}^{i_{l}}}>0
$$

and we pass to the next $\Omega_{k}^{i_{l^{\prime}}}$, if there is any, where $i_{l^{\prime}} \in I_{0}$, or we have

$$
\lim _{k \rightarrow+\infty} \frac{\rho_{k}^{i_{l}}}{R_{k}^{i_{l}}}=0
$$

In such a case we apply again the energy tracking lemma III.2 on the annulus $B_{R_{k}^{i_{l}} / 2}(x) \backslash B_{\rho_{k}^{i_{l}}}(x)$ where $x \in \Omega_{k}^{i_{l}}$ satisfies

$$
\int_{B_{\rho_{k}}(x)}\left|d \vec{n}_{\vec{\xi}_{k}}\right|_{g_{k}}^{2} d v o l_{g_{k}}=\varepsilon_{0}
$$

and we set $x_{k}^{i, 2}:=x$ and $\rho_{k}^{i, 2}:=\rho_{k}^{i_{l}}$. We keep this procedure running until all annuli of the type $I_{0}$ have been explored, and until the neighborhood of each $a^{i}$ has been considered. This can be done in a finite number of steps, since each application of lemma III.2 takes place around a "bubble" exhausting at least $\varepsilon_{0}$ of the total energy of the sequence $\vec{\xi}_{k}$. With moderate effort, one checks that the sequence of finite families of bubbles constructed at the end of the procedure fulfills the desired requirements of proposition

\section{Construction of a Coulomb moving frame in each component of the neck region}

Let $\vec{\xi}_{k}$ be a family of weakly converging Willmore immersions of $\Sigma$ fulfilling the conclusions of lemma II.1. For some $\varepsilon_{0}$ chosen smaller than the constant $\varepsilon$ appearing in the $\varepsilon$-regularity theorem I.5 in [Ri2, we apply proposition III.1 so as to obtain a subsequence for which we can decompose the surface into "converging regions" (complements of small neighborhoods of the points $a^{i}$ ), bubbles, and neck regions. In each annulus $B_{R_{k}}\left(x_{k}^{i, j}\right) \backslash B_{r_{k}}\left(x_{k}^{i, j}\right) \subset \Omega_{k}(\alpha)$ of a part of the neck region $\Omega_{k}(\alpha)$ satellite to some bubble centered on $x_{k}^{i, j}$, the condition (III.13) states that

$$
\sup _{r_{k}<\rho<R_{k}} \int_{B_{2 \rho}\left(x_{k}^{i, j}\right) \backslash B_{\rho}\left(x_{k}^{i, j}\right)}\left|\nabla \vec{n}_{\vec{\xi}_{k}}\right| d x<\varepsilon_{0} .
$$

Combining this fact with the $\varepsilon$-regularity theorem I.5 in Ri2] gives that

$$
\forall x \in B_{R_{k} / 2}\left(x_{k}^{i, j}\right) \backslash B_{2 r_{k}}\left(x_{k}^{i, j}\right) \quad\left|\nabla \vec{n}_{\vec{\xi}_{k}}\right| \leq C_{m} \frac{\sqrt{\varepsilon_{0}}}{\left|x-x_{k}^{i, j}\right|} .
$$

This pointwise estimate translates into a control of the $L^{2}$-weak norm of $\nabla \vec{n}_{\vec{\xi}}$ in the annulus, namely:

$$
\left\|\nabla \vec{n}_{\vec{\xi}_{k}}\right\|_{L^{2, \infty}\left(B_{R_{k} / 2}\left(x_{k}^{i, j}\right) \backslash B_{2 r_{k}}\left(x_{k}^{i, j}\right)\right)} \leq C_{m} \sqrt{\varepsilon_{0}}
$$


This $L^{2}$-weak estimate for the gradient of the Gauss map in neck regions already appears in previous works LiRi1, LiRi2 and [Ri5], where it plays a decisive role. It is also of utmost importance in the present paper, as it will enable us in lemma IV.3 to construct a $W^{1,2}$ Coulomb gauge without having to resort to the stronger $L^{2}$-control of the gradient of the Gauss map commonly required in similar situations. Our results are generic in nature, and they should be helpful to the study of other gauge invariant problems.

We first need an extension lemma.

Lemma IV.1 Let $\vec{n} \in W^{1,2}\left(B_{2}(0) \backslash B_{1}(0), G_{m-2}\left(\mathbb{R}^{m}\right)\right)$ where $G_{m-2}\left(\mathbb{R}^{m}\right)$ denotes the Grassmannian manifold of oriented $(m-2)$-planes in $\mathbb{R}^{m}$. There exists $\delta(m)>0$ depending only on $m$ such that if

$$
\int_{\partial B_{1}(0)}|\nabla \vec{n}| d \theta \leq \delta(m)
$$

then we can extend $\vec{n}$ to $\hat{\vec{n}} \in W^{1,2}\left(B_{2}(0), G_{m-2}\left(\mathbb{R}^{m}\right)\right)$ with $\hat{\vec{n}}=\vec{n}$ on the annulus $B_{2}(0) \backslash B_{1}(0)$, and furthermore

$$
\int_{B_{2}(0)}|\nabla \hat{\vec{n}}|^{2} d x \leq C_{m} \int_{B_{2}(0) \backslash B_{1}(0)}|\nabla \vec{n}|^{2} d x \quad,
$$

where $C_{m}>0$ depends only on $m$.

Proof of lemma IV.1. Suppose that

$$
\int_{\partial B_{1}(0)}|\nabla \vec{n}| d \theta \leq \delta
$$

for some $\delta>0$. Then

$$
\|\vec{n}-\vec{n}(1,0)\|_{L^{\infty}\left(\partial B_{1}(0)\right)} \leq \int_{\partial B_{1}(0)}\left|\frac{\partial \vec{n}}{\partial \theta}\right| d \theta \leq \delta \quad .
$$

Thus, if $\delta>0$ chosen small enough and independently of $\vec{n}(1,0)$, the plane $\vec{n}(p)$ is contained in a small geodesic ball $B_{\delta}(\vec{n}(1,0)) \subset G_{m-2}\left(\mathbb{R}^{m}\right)$ on which coordinates $y=\left(y_{1}, \ldots, y_{N}\right)$ exist. We may further choose these coordinates in such a way that $B_{\delta}(\vec{n}(1,0))$ corresponds to the ball $B_{\delta}^{N}(0)$ of radius $\delta$ and centered on $0 \in \mathbb{R}^{N}$. Owing to the compactness of $G_{m-2}\left(\mathbb{R}^{m}\right)$, we may always arrange for $\left\|\nabla y^{-1}\right\|_{L^{\infty}\left(B_{\delta}(0)\right)}$ to depend only on $m$. We have 8

$$
\|\vec{n}\|_{H^{1 / 2}\left(\partial B_{1}(0)\right)} \simeq \sum_{l=1}^{N}\left\|n_{l}\right\|_{H^{1 / 2}\left(\partial B_{1}(0)\right)}
$$

where $n_{l}$ are the coordinates of $\vec{n}$ in the system $\left\{y_{l}\right\}_{l=1, \ldots, N}$. Let $\hat{n}_{l}$ be the harmonic extension of $n_{l}$ into $B_{1}(0)$. Since the $\mathbb{R}^{N}$-valued map $\left(n_{l}\right)$ is included in the ball $B_{\delta}^{N}(0)$, the maximum principle yields that $\left(\hat{n}_{l}\right)_{l=1, \ldots, N} \in B_{\delta}^{N}(0)$. Moreover,

$$
\int_{B_{1}(0)}\left|\nabla \hat{n}_{l}\right|^{2} \leq C \sum_{l=1}^{N}\left\|n_{l}\right\|_{H^{1 / 2}\left(\partial B_{1}(0)\right)}^{2} \leq c_{m}\|\vec{n}\|_{H^{1 / 2}\left(\partial B_{1}(0)\right)}^{2} \leq C_{m} \int_{B_{2}(0) \backslash B_{1}(0)}|\nabla \vec{n}|^{2},
$$

where the constants $c_{m}$ and $C_{m}$ depend only on $m$. Note that in the last inequality, we have again used the characterization of $\vec{n}$ as an $\mathbb{R}^{K_{m}}$-valued map. We set $\hat{\vec{n}}:=y^{-1}\left(\left(\hat{n}_{l}\right)\right)$. Since $\left\|\nabla y^{-1}\right\|_{L^{\infty}\left(B_{\delta}(0)\right)}$

\footnotetext{
${ }^{8}$ On the left-hand side of (IV.6), we use the isometric embedding of $G_{m-2}\left(\mathbb{R}^{m}\right)$ into some $\mathbb{R}^{K_{m}}$, and $\vec{n}$ is viewed as an $\mathbb{R}^{K_{m}}$-valued map.
} 
only depends on $m$, (IV.7) implies the desired (IV.4), thereby proving lemma IV.1 with $\delta(m)=\delta$ small enough.

The main result of this section also requires an integrability-by-compensation lemma for second-order equations in divergence form. It was originally proved by Y. Ge in Ge through a different method from the one used here.

Lemma IV.2 [Wente estimates in Lorentz $L^{p, q}$ spaces] Consider the divergence-form problem

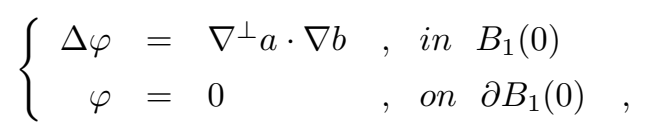

where $\nabla a \in L^{2, \infty}\left(B_{1}(0)\right)$, and $\nabla b \in L^{p, q}\left(B_{1}(0)\right)$ for some $p \in(1, \infty)$ and $q \in[1, \infty]$. Then there holds

$$
\|\nabla \varphi\|_{L^{p, q}\left(B_{1}(0)\right)} \leq C_{p, q}\|\nabla a\|_{L^{2, \infty}\left(B_{1}(0)\right)}\|\nabla b\|_{L^{p, q}\left(B_{1}(0)\right)}
$$

for some constant $C_{p, q}>0$.

Proof of lemma IV.2, Owing to the interpolative nature of the Lorentz space $L^{p, q}$, it suffices to show that (IV.9) holds for $p=q \in(1, \infty)$. Consider first the case when $p>2$. According to the Hölder inequality for Lorentz spaces, there holds

$$
L^{2, \infty} \cdot L^{p} \subset L^{\frac{2 p}{2+p}, p}
$$

Since $p>2$, we call upon the usual Calderon-Zygmund theorem and the Sobolev embedding theorem to obtain that $\nabla \varphi \in W^{1, \frac{2 p}{2+p}} \subset L^{p}$ with the estimate (IV.9), as claimed.

Next, when $1<p \leq 2$, we use the divergence-form structure of the equation. Note that

$$
\Delta \varphi=\operatorname{div}\left(b \nabla^{\perp} a\right)
$$

and that

$$
b \nabla^{\perp} a \in W^{1, p} \cdot L^{2, \infty} \subset L^{\frac{2 p}{2-p}, p} \cdot L^{2, \infty} \subset L^{p},
$$

where we have again used the Sobolev embedding theorem and the Hölder inequality for Lorentz spaces. The desired (IV.9) follows immediately from (IV.11) and (IV.12) and this concludes the proof of lemma IV.2.

We next state and prove the main result of this section.

Lemma IV.3 $\left[W^{1,2}\right.$-controlled Coulomb frame from small $L^{2, \infty}$-control of the second fundamental form] Let $\vec{n} \in W^{1,2}\left(B_{1}(0) \backslash B_{r}(0), G_{m-2}\left(\mathbb{R}^{m}\right)\right)$ where $0 \leq r<1 / 2$. There exists $\eta(m)>0$ depending only on $m$ (but not on $r$ ) such that if

$$
\|\nabla \vec{n}\|_{L^{2, \infty}\left(B_{1}(0)\right)} \leq \eta(m)
$$

and if

$$
\int_{\partial B_{r}(0)}|\nabla \vec{n}| d l_{\partial B_{r}} \leq \eta(m),
$$

where $d l_{\partial B_{r}}$ is the length form $r d \theta$ on $\partial B_{r}$, then one can construct an associated Coulomb frame $\left\{\vec{e}_{1}, \vec{e}_{2}\right\}$ satisfying

$$
\star \vec{n}=\vec{e}_{1} \wedge \vec{e}_{2} \quad \text { and } \quad \operatorname{div}\left(\vec{e}_{1} \cdot \nabla \vec{e}_{2}\right)=0
$$




\section{Furthermore,}

$$
\int_{B_{1}(0) \backslash B_{r}(0)}\left|\nabla \vec{e}_{1}\right|^{2}+\left|\nabla \vec{e}_{2}\right|^{2} \leq C \int_{B_{1}(0) \backslash B_{r}(0)}|\nabla \vec{n}|^{2}
$$

and

$$
\sum_{i=1}^{2}\left\|\nabla \vec{e}_{i}\right\|_{L^{2, \infty}\left(B_{1}(0) \backslash B_{r}(0)\right)} \leq C\|\nabla \vec{n}\|_{L^{2, \infty}\left(B_{1}(0) \backslash B_{r}(0)\right)},
$$

for some constant $C$ depending on $m$, but neither on $r$ nor on $\vec{n}$.

Proof of lemma IV.3. Owing to lemma IV.1, we can always reduce to the case when $r=0$. Indeed, we have seen that the extension $\hat{\vec{n}}$ in $B_{r}(0)$ of the restriction of $\vec{n}$ to $B_{2 r}(0) \backslash B_{r}(0)$ satisfies

$$
\int_{B_{r}(0)}|\nabla \hat{\vec{n}}|^{2} d x \leq C_{m} \int_{B_{2 r}(0) \backslash B_{r}(0)}|\nabla \vec{n}|^{2} d x \leq C_{m} \eta(m)
$$

In particular, if lemmaIV.1 holds for $r=0$ with the constant $\eta(m)>0$, then it also holds for an arbitrary $0<r<1 / 2$ with the constant $\eta(m) /\left(C_{m}+1\right)$ playing the role of $\eta(m)$ in the statement. We shall thus focus on the case $r=0$.

We first suppose that $\vec{n}$ is smooth. According to Theorem 5.2.1 in [Hel], there exists a smooth moving orthonormal two-frame $\left\{\vec{f}_{1}, \vec{f}_{2}\right\}$ in $W^{1,2}\left(B_{1}(0), \mathbb{R}^{m}\right)$ with $\star \vec{n}=\vec{f}_{1} \wedge \overrightarrow{f_{2}}$. Its energy is however not controlled, since we do not assume that $\|\nabla \vec{n}\|_{L^{2}\left(B_{1}(0)\right)}$ is smaller than the required threshold $8 \pi / 3$.

For each $r \in[0,1]$, let $\left\{\vec{f}_{1, r}(x), \vec{f}_{2, r}(x)\right\}:=\left\{\vec{f}_{1}(r x), \vec{f}_{2}(r x)\right\}$. We minimize

$$
F_{r}\left(\theta_{r}\right):=\int_{B_{r}(0)}\left|\nabla \theta_{r}+\left(\vec{f}_{1, r} \cdot \nabla \vec{f}_{2, r}\right)\right|^{2}
$$

over all rotations $\theta_{r} \in W^{1,2}\left(B_{1}(0), \mathbb{R}\right)$. As explained in Theorem 4.1.1 from Hel, for each $r$, the minimum of $F_{r}$ is attained at some frame $\left\{\vec{e}_{1, r}, \vec{e}_{2, r}\right\}$ satisfying

$$
\vec{e}_{1, r}+i \vec{e}_{2, r}=e^{i \theta_{r}}\left(\vec{f}_{1, r}+i \vec{f}_{2, r}\right)
$$

and

$$
\left\{\begin{array}{l}
\operatorname{div}\left(\vec{e}_{1, r} \cdot \nabla \vec{e}_{2, r}\right)=0 \quad \text { on } B_{r}(0) \\
\vec{e}_{1, r} \cdot \partial_{\nu} \vec{e}_{2, r}=0 \quad \text { on } \partial B_{r}(0) .
\end{array}\right.
$$

Thus, there exists $\lambda_{r}$ equal to zero on $\partial B_{r}(0)$ and solving

$$
\nabla \lambda_{r}=-\vec{e}_{1, r} \cdot \nabla^{\perp} \vec{e}_{2, r}
$$

It satisfies in particular

$$
\left\{\begin{array}{rlrl}
\Delta \lambda_{r} & =\nabla^{\perp} \vec{e}_{1, r} \cdot \nabla \vec{e}_{2, r} & & \text { in } B 2_{r}(0) \\
\lambda_{r} & =0 & & \text { on } \partial B_{r}(0)
\end{array} .\right.
$$

Using the Wente inequality lemma IV.2 with $(p, q)=(2, \infty)$ yields

$$
\left\|\nabla \lambda_{r}\right\|_{L^{2, \infty}\left(B_{r}(0)\right)} \leq C_{0}\left\|\nabla \vec{e}_{1, r}\right\|_{L^{2, \infty}\left(B_{r}(0)\right)}\left\|\nabla \vec{e}_{2, r}\right\|_{L^{2, \infty}\left(B_{r}(0)\right)},
$$

for some constant $C_{0}$ independent of $r$. Moreover, as shown in the proof of Lemma 5.1.4 in [Hel], an elementary computation gives

$$
\left|\nabla \vec{e}_{1, r}\right|^{2}+\left|\nabla \vec{e}_{2, r}\right|^{2}=2\left|\nabla \lambda_{r}\right|^{2}+|\nabla \vec{n}|^{2} .
$$


Combining altogether (IV.21) and (IV.22) gives the inequality

$$
\left\|\nabla \lambda_{r}\right\|_{L^{2, \infty}\left(B_{r}(0)\right)}^{2}+\frac{1}{4 C_{0}}\left(C_{0}\|\nabla \vec{n}\|_{L^{2, \infty}}-1\right)\left\|\nabla \lambda_{r}\right\|_{L^{2, \infty}\left(B_{r}(0)\right)}+C_{0}\|\nabla \vec{n}\|_{L^{2, \infty}\left(B_{r}(0)\right)} \geq 0 \quad .
$$

Accordingly, there exists some small enough threshold $\eta\left(C_{0}\right) \equiv \eta(m)$ for which the hypothesis that $\|\nabla \vec{n}\|_{L^{2, \infty}\left(B_{1}(0)\right)}<\eta(m)$ guarantees that the range of $\left\|\nabla \lambda_{r}\right\|_{L^{2, \infty}\left(B_{r}(0)\right)}$ is of the form $[0, \alpha(\eta)] \cup[\beta(\eta), \infty)$ for some $0<\alpha(\eta)<\beta(\eta)$. One easily checks from (IV.23) that $\alpha(\eta) \leq C_{1}\|\nabla \vec{n}\|_{L^{2, \infty}\left(B_{1}(0)\right)}$ with the constant $C_{1}$ depending only on $C_{0}$, and thus only on $m$.

As $\vec{f}_{j, r}(x)=\vec{f}_{j}(r x)$ and $\vec{f}_{j}$ belongs to $W^{1,2}\left(B_{1}(0)\right)$, it is clear that $\vec{f}_{j, r}$ is continuous in the parameter $r$. From this and the definition of $\theta_{r}$, it follows that $\theta_{r}$ is likewise continuous in $r$, and hence from (IV.18) and IV.20), that $\vec{e}_{j, r}$ and $\lambda_{r}$ are continuous in $r$. We see that $\left\|\nabla \lambda_{r}\right\|_{L^{2, \infty}\left(B_{r}(0)\right)}$ takes the value 0 at $r=0$. From the above discussion, we deduce that at $r=1$,

$$
\|\nabla \lambda\|_{L^{2, \infty}\left(B_{1}(0)\right)} \equiv\left\|\nabla \lambda_{1}\right\|_{L^{2, \infty}\left(B_{1}(0)\right)} \leq \alpha(\eta) \leq C_{1}\|\nabla \vec{n}\|_{L^{2, \infty}\left(B_{1}(0)\right)} .
$$

Let now $\left\{\vec{e}_{1}, \vec{e}_{2}\right\}:=\left\{\vec{e}_{1,1}, \vec{e}_{2,1}\right\}$. By definition, this Coulomb frame satisfies

$$
\star \vec{n}=\vec{e}_{1} \wedge \vec{e}_{2} \quad, \quad \operatorname{div}\left(\vec{e}_{1} \cdot \nabla \vec{e}_{2}\right)=0 \quad, \quad \nabla^{\perp} \lambda=\vec{e}_{1} \cdot \nabla \vec{e}_{2}=-\vec{e}_{2} \cdot \nabla \vec{e}_{1}
$$

Note next that

$$
\left|\nabla \vec{e}_{j}\right|^{2}=\left|\vec{e}_{j^{\prime}} \cdot \nabla \vec{e}_{j}\right|^{2}+\left|\pi_{\vec{n}} \nabla \vec{e}_{j}\right|^{2} \leq|\nabla \lambda|^{2}+|\nabla \vec{n}|^{2}, \quad \text { for } \quad\left(j, j^{\prime}\right) \in\{(1,2),(2,1)\} \quad .
$$

In particular using (IV.24), we find

$$
\left\|\nabla \vec{e}_{j}\right\|_{L^{2, \infty}\left(B_{1}(0)\right)} \leq\|\nabla \lambda\|_{L^{2, \infty}\left(B_{1}(0)\right)}+\|\nabla \vec{n}\|_{L^{2, \infty}\left(B_{1}(0)\right)} \leq\left(C_{1}+1\right)\|\nabla \vec{n}\|_{L^{2, \infty}\left(B_{1}(0)\right)},
$$

thereby giving the required (IV.16).

Calling again upon the Wente inequality of lemma IV.2 with $(p, q)=(2,2)$, we obtain from (IV.20) with $r=1$ that

$$
\begin{aligned}
\|\nabla \lambda\|_{L\left(B_{1}(0)\right)} & \leq C_{2}\left\|\nabla \vec{e}_{1}\right\|_{L^{2, \infty}\left(B_{1}(0)\right)}\left\|\nabla \vec{e}_{2}\right\|_{L\left(B_{1}(0)\right)} \\
& \leq C_{2}\left(C_{1}+1\right) \eta(m)\left(\|\nabla \lambda\|_{L\left(B_{1}(0)\right)}+\|\nabla \vec{n}\|_{L\left(B_{1}(0)\right)}\right),
\end{aligned}
$$

where we have used (IV.26), (IV.27), and the hypothesis (IV.13). Here $C_{2}$ is a constant that depends only on $m$. The latter shows that $\|\nabla \lambda\|_{L\left(B_{1}(0)\right)} \leq C_{3} \eta(m)\|\nabla \vec{n}\|_{L\left(B_{1}(0)\right)}$, which, once introduced into (IV.26) gives the announced

$$
\int_{B_{1}(0)}\left|\nabla \vec{e}_{1}\right|^{2}+\left|\nabla \vec{e}_{2}\right|^{2} \leq C \int_{B_{1}(0)}|\nabla \vec{n}|^{2}
$$

where the constant $C$ depends only on $m$.

In the general case when $\vec{n}$ is not smooth, we use the limiting process outlined in step 6 of the proof of Lemma 5.1.4 from [Hel], and based on the known fact $[\mathrm{ScU}]$ that $C^{\infty}\left(B_{1}(0), G_{m-2}\left(\mathbb{R}^{m}\right)\right)$ is dense into $W^{1,2}\left(B_{1}(0), G_{m-2}\left(\mathbb{R}^{m}\right)\right)$. This concludes the proof of lemma IV.3.

\section{General estimates in the neck regions of weakly converging weak conformal immersions}

Lemma V.1 [Estimate of the "Euler characteristic production" in neck regions] There exists a constant $\eta(m)>0$ with the following property. Let $\eta<\eta(m)$, let $R>4 r>0$, and let $\vec{\xi}$ be a conformal weak immersion from $B_{R}(0) \backslash B_{r}(0)$ into $\mathbb{R}^{m}$ with $L^{2}$-bounded second fundamental form satisfying

$$
\left\|\nabla \vec{n}_{\vec{\xi}}\right\|_{L^{2, \infty}\left(B_{R} \backslash B_{r}\right)} \leq \eta \quad,
$$




$$
\int_{\partial B_{r}(0)}\left|\nabla \vec{n}_{\vec{\xi}}\right| d l_{\partial B_{r}(0)} \leq \eta
$$

and

$$
\int_{B_{R} \backslash B_{R / 2} \cup B_{2 r} \backslash B_{r}}\left|\nabla \vec{n}_{\vec{\xi}}\right|^{2} d x \leq \eta
$$

Then there exists a positive constant $C_{m}$ depending only on $m$, but not on the conformal type of the annulus $\log \frac{R}{r}$, such that

$$
\left|\int_{B_{R}(0) \backslash B_{r}(0)} K_{\vec{\xi}} d v o l_{g_{\vec{\xi}}}\right| \leq C_{m} \eta
$$

where $K_{\vec{\xi}}$ denotes the Gaussian curvature associated with the immersion $\vec{\xi}$.

Proof of lemma V.1, Let $\Omega=B_{R}(0) \backslash B_{r}(0)$. According to lemma IV.3 (applicable here owing to the hypotheses (V.1) and ( $(\overline{V .2})$ ), there exists a Coulomb moving frame

$$
\left\{\vec{e}_{1}, \vec{e}_{2}\right\} \in\left(W^{1,2}\left(\Omega, S^{m-1}\right)\right)^{2}
$$

satisfying

$$
\star \vec{n}_{\vec{\xi}}=\vec{e}_{1} \wedge \vec{e}_{2}
$$

and

$$
\sum_{i=1}^{2}\left\|\nabla \vec{e}_{i}\right\|_{L^{2, \infty}(\Omega)} \leq C_{1}\|\nabla \vec{n}\|_{L^{2, \infty}(\Omega)} \leq C_{2} \eta
$$

where the constants $C_{j}$ depend only on $m$. The mean value theorem yields the existence of some $\alpha \in$ $(1 / 2,1)$ with

$$
\begin{aligned}
\sum_{i=1}^{2} \int_{\partial\left(B_{\alpha R} \backslash B_{\alpha}-1_{r}\right)}\left|\nabla \vec{e}_{i}\right| & \leq C_{3} r \sum_{i=1}^{2}\left\|\nabla \vec{e}_{i}\right\|_{L^{1}\left(B_{2 r} \backslash B_{r}\right)}+C_{4} R \sum_{i=1}^{2}\left\|\nabla \vec{e}_{i}\right\|_{L^{1}\left(B_{R} \backslash B_{R / 2}\right)} \\
& \leq C_{5} \sum_{i=1}^{2}\left\|\nabla \vec{e}_{i}\right\|_{L^{2, \infty}(\Omega)} \leq C_{5} C_{2} \eta
\end{aligned}
$$

where $C_{5}$ is a universal constant.

The Liouville equation states that

$$
\Delta \lambda=-e^{2 \lambda} K_{\vec{\xi}},
$$

where $\lambda$ is the conformal parameter of the conformal immersion $\vec{\xi}$. From the way the Coulomb frame $\left\{\vec{e}_{1}, \vec{e}_{2}\right\}$ was constructed, there also holds on the annulus $B_{R}(0) \backslash B_{r}(0)$ :

$$
\Delta \lambda=-\nabla \vec{e}_{1} \cdot \nabla^{\perp} \vec{e}_{2}=-\operatorname{div}\left(\vec{e}_{1} \cdot \nabla^{\perp} \vec{e}_{2}\right) .
$$

Hence, we find using (V.6)

$$
\begin{aligned}
& \left|\int_{B_{\alpha R}(0) \backslash B_{\alpha^{-1} r_{r}(0)}} K_{\vec{\xi}} e^{2 \lambda} d x\right|=\left|\int_{B_{\alpha R}(0) \backslash B_{\alpha^{-1} r_{r}(0)}} \nabla \vec{e}_{1} \cdot \nabla^{\perp} \vec{e}_{2} d x\right| \\
& \leq \int_{\partial B_{\alpha R}(0)}\left|e_{1} \cdot \partial_{\tau} e_{2}\right| d l+\int_{\partial B_{\alpha^{-1} r_{r}(0)}}\left|e_{1} \cdot \partial_{\tau} e_{2}\right| d l \leq C \eta \quad,
\end{aligned}
$$


where $\tau$ denotes the unit tangent vector to the circles $\partial B_{\rho}(0)$, namely $\tau=\rho^{-1} \partial_{\theta}$.

Moreover, owing to the hypothesis $(\overline{V .3})$ and the fact that $\alpha \in(1 / 2,1)$, we have that

$$
\int_{B_{\alpha^{-1} r}(0) \backslash B_{r}(0)}\left|K_{\vec{\xi}}\right| e^{2 \lambda} d x \leq 2^{-1} \int_{B_{\alpha^{-1}}(0) \backslash B_{r}(0)}\left|\nabla \vec{n}_{\vec{\xi}}\right|^{2} d x \leq 2^{-1} \eta \quad,
$$

and similarly

$$
\int_{B_{R}(0) \backslash B_{\alpha R}(0)}\left|K_{\vec{\xi}}\right| e^{2 \lambda} d x \leq 2^{-1} \int_{B_{R}(0) \backslash B_{\alpha R}(0)}\left|\nabla \vec{n}_{\vec{\xi}}\right|^{2} d x \leq 2^{-1} \eta .
$$

Altogether (V.9), (V.10), and (V.11) yield the announced (V.4).

Lemma V.2 [Uniform Harnack estimates for the conformal factor in neck regions] There exists a constant $\varepsilon(m)>0$ with the following property. Let $1<R<\infty$. If $\vec{\xi}$ is a Lipschitz conformal immersion from $\Omega_{R}:=B_{R}(0) \backslash B_{R^{-1}}(0)$ into $\mathbb{R}^{m}$ satisfying

$$
\sup _{r \in\left(R^{-1}, R / 2\right)} \int_{B_{2 r}(0) \backslash B_{r}(0)}\left|\nabla \vec{n}_{\vec{\xi}}\right|^{2} d x \leq \varepsilon(m),
$$

and

$$
\|\nabla \lambda\|_{L^{2, \infty}\left(\Omega_{R}\right)}=A<+\infty .
$$

Then for any $4 R^{-2}<\alpha<1$ there exists $C_{\alpha}(A)>0$, depending only on $\alpha$ and $A$ but not on $R$, such that

$$
\sup _{r \in\left(4 R^{-1}, \alpha R\right)} \frac{\sup _{x \in B_{\alpha^{-1} r_{r}}(0) \backslash B_{r}(0)} e^{\lambda(x)}}{\inf _{x \in B_{\alpha^{-1}}(0) \backslash B_{r}(0)} e^{\lambda(x)}} \leq C_{\alpha} \quad .
$$

Proof of lemma V.2. Let $r \in\left(4 R^{-1}, R / 4\right)$. From (V.12), we have

$$
\int_{B_{3 r}(0) \backslash B_{r / 4}(0)}\left|\nabla \vec{n}_{\vec{\xi}}\right|^{2} d x \leq 4 \varepsilon(m) .
$$

With the help of the Fubini and mean value theorems, we deduce from (V.12) the existence of $\rho \in$ $(r / 4, r / 2)$ such that

$$
\int_{\partial B_{\rho}(0)}\left|\nabla \vec{n}_{\vec{\xi}}\right|^{2} \leq \frac{4}{r} \varepsilon(m) \leq \frac{2}{\rho} \varepsilon(m)
$$

hence

$$
\int_{\partial B_{\rho}(0)}\left|\nabla \vec{n}_{\vec{\xi}}\right| d x \leq \sqrt{4 \pi \varepsilon(m)} .
$$

Following lemma IV.1, we choose $\sqrt{4 \pi \varepsilon(m)}<\delta(m)$ so as to obtain an extension $\hat{\vec{n}}$ of $\vec{n}$ in $B_{\rho}(0)$ with

$$
\int_{B_{4 r}(0)}|\nabla \hat{\vec{n}}|^{2} d x \leq 4 C_{m} \varepsilon(m)
$$

where $C_{m}$ is the constant depending only on $m$ in the statement of lemma IV.1. Insuring further that $12 C_{m} \varepsilon_{m} \leq 8 \pi$, we can apply lemma 5.1.4 of [Hel] in order to find a framing $\left\{\vec{e}_{1}, \vec{e}_{2}\right\} \in\left(W^{1,2}\left(B_{4 r}(0)\right)\right)^{2}$ satisfying $\star \hat{\vec{n}}=\vec{e}_{1} \wedge \vec{e}_{2}$ and

$$
\int_{B_{4 r}(0)} \sum_{i=1}^{2}\left|\nabla \vec{e}_{i}\right|^{2} d x \leq C \int_{B_{4 r}(0)}|\nabla \hat{\vec{n}}|^{2} d x \leq 4 C C_{m} \varepsilon(m) .
$$

\footnotetext{
${ }^{9}$ as before, $\lambda$ is the conformal parameter of $\vec{\xi}$. Namely, $\left|\partial_{x_{1}} \vec{\xi}\right|=e^{\lambda}=\left|\partial_{x_{2}} \vec{\xi}\right|$.
} 
The conformal parameter satisfies

$$
\Delta \lambda=\nabla^{\perp} \vec{e}_{1} \cdot \nabla \vec{e}_{2} \quad \text { in } B_{4 r}(0) \backslash B_{r / 2}(0) .
$$

Let $\mu$ be the solution of

$$
\begin{cases}\Delta \mu=\nabla^{\perp} \vec{e}_{1} \cdot \nabla \vec{e}_{2} & \text { in } B_{4 r}(0) \backslash B_{r / 2}(0) \\ \mu=0 & \text { on } \partial\left(B_{4 r}(0) \backslash B_{r / 2}(0)\right)\end{cases}
$$

A standard Wente estimate (see theorem 3.3.8 in [Hel]) yields on one hand

$$
\|\mu\|_{L^{\infty}\left(B_{4 r}(0) \backslash B_{r / 2}(0)\right)} \leq(2 \pi)^{-1}\left\|\nabla \vec{e}_{1}\right\|_{L^{2}}\left\|\nabla \vec{e}_{2}\right\|_{L^{2}} \leq C \varepsilon(m),
$$

and on the other hand

$$
\|\nabla \mu\|_{L\left(B_{4 r}(0) \backslash B_{r / 2}(0)\right)} \leq \sqrt{3 / 32 \pi}\left\|\nabla \vec{e}_{1}\right\|_{L^{2}}\left\|\nabla \vec{e}_{2}\right\|_{L^{2}} \leq C \varepsilon(m) \quad .
$$

Let $\nu:=\lambda-\mu$ be the harmonic function in $B_{4 r}(0) \backslash B_{r / 2}(0)$ equal to $\lambda$ on the boundary. Owing to (V.13) and (V.23), there holds

$$
\|\nabla \nu\|_{L^{2, \infty}\left(B_{4 r}(0) \backslash B_{r / 2}(0)\right)} \leq C \varepsilon(m)+A \quad .
$$

Let $\bar{\nu}$ be the average of $\nu$ on $\left.B_{4 r}(0) \backslash B_{r / 2}(0)\right)$. The Sobolev-Poincaré inequality gives

$$
\begin{aligned}
r^{-2} \int_{B_{4 r}(0) \backslash B_{r / 2}(0)}|\nu-\bar{\nu}| d x & \leq C_{1} r^{-1} \int_{B_{4 r}(0) \backslash B_{r / 2}(0)}|\nabla \nu| d x \\
& \leq C_{2}\|\nabla \nu\|_{L^{2, \infty}\left(B_{4 r}(0) \backslash B_{r / 2}(0)\right)} \leq C_{3} \varepsilon(m)+C_{2} A
\end{aligned}
$$

where $C_{j}$ are universal constants. Since $\nu-\bar{\nu}$ is harmonic, we deduce that on a smaller annulus the $L^{\infty}$-norm of $(\nu-\bar{\nu})$ is controled by $\varepsilon(m)$ and $A$ :

$$
\|\nu-\bar{\nu}\|_{L^{\infty}\left(B_{2 r}(0) \backslash B_{r}(0)\right.} \leq C r^{-2} \int_{B_{4 r}(0) \backslash B_{r / 2}(0)}|\nu-\bar{\nu}| d x_{1} d x_{2} \leq C_{4}(\varepsilon(m)+A),
$$

where $C_{4}$ is a universal constant. We set $\lambda_{-}:=\inf \left\{\lambda(x) ; x \in B_{2 r}(0) \backslash B_{r}(0)\right\}, \lambda_{+}:=\max \{\lambda(x) ; x \in$ $\left.B_{2 r}(0) \backslash B_{r}(0)\right\}, \nu_{-}:=\inf \left\{\nu(x) ; x \in B_{2 r}(0) \backslash B_{r}(0)\right\}$, and $\nu_{+}:=\max \left\{\nu(x) ; x \in B_{2 r}(0) \backslash B_{r}(0)\right\}$. One has the estimates

$$
\frac{e^{\lambda_{+}}}{e^{\lambda_{-}}} \leq \frac{e^{\nu_{+}+\|\mu\|_{\infty}}}{e^{\nu_{-}-\|\mu\|_{\infty}}} \leq e^{2\|\mu\|_{\infty}} e^{\nu_{+}-\nu_{-}} \leq e^{2 C \varepsilon(m)} e^{2 C_{4}(\varepsilon(m)+A)}
$$

where we have used respectively (V.22) and (V.26).

We have thus proved (V.14) for $\alpha=1 / 2$. Let now $\alpha=2^{-j}$, for some $j \in \mathbb{N}^{*}$. The idea is to apply (V.14) successively $j$ times, with $\alpha=1 / 2$ and with $2^{j} r$ in place of $r$. For $i=1, \ldots, j$, we let $\lambda_{-}^{i}:=\inf \left\{\lambda(x) ; x \in B_{2^{i} r}(0) \backslash B_{2^{i-1} r}(0)\right\}$ and $\lambda_{+}^{i}:=\max \left\{\lambda(x) ; x \in B_{2^{i} r}(0) \backslash B_{2^{i-1} r}(0)\right\}$. Using the fact that

$$
\lambda_{-}^{i} \leq \lambda_{+}^{i \pm 1}
$$

gives

$$
\frac{e^{\lambda_{+}^{i}}}{e^{\lambda_{-}^{i \pm 1}}} \leq \frac{e^{\lambda_{+}^{i}}}{e^{\lambda_{-}^{i}}} \frac{e^{\lambda_{-}^{i}}}{e^{\lambda_{+}^{i \pm 1}}} \frac{e^{\lambda_{+}^{i \pm 1}}}{e^{\lambda_{-}^{i \pm 1}}} \leq \frac{e^{\lambda_{+}^{i}}}{e^{\lambda_{-}^{i}}} \frac{e^{\lambda_{+}^{i \pm 1}}}{e^{\lambda_{-}^{i \pm 1}}}
$$

The latter enables an iteration, and eventually yields that (V.14) holds with $\alpha=2^{-j}$, for an arbitrary positive integer $j$ satisfying $4 R^{-2}<2^{-j}$, thereby concluding the proof of Lemma V.2. 
Lemma V.3 [Pointwise control of the conformal factor in the neck region] There exists a constant $\eta(m)>0$ with the following property. Let $0<\eta<\eta(m)$ and $0<4 r<R<+\infty$. If $\vec{\xi}$ is any (weak) conformal immersion of $\Omega:=B_{R}(0) \backslash B_{r}(0)$ into $\mathbb{R}^{m}$ with $L^{2}$-bounded second fundamental form, and satisfying

$$
\left\|\nabla \vec{n}_{\vec{\xi}}\right\|_{L^{2, \infty}(\Omega)} \leq \sqrt{\eta}
$$

then there exist $\alpha \in(1 / 2,1)$ and $A \in \mathbb{R}$ (depending on $R, r, m$, and $\vec{\xi}$ ) such that

$$
\|\lambda(x)-d \log |x|-A\|_{L^{\infty}\left(B_{\alpha R}(0) \backslash B_{\alpha^{-1} r}(0)\right)} \leq C_{m}\left(\|\nabla \lambda\|_{L^{2, \infty}(\Omega)}+\int_{\Omega}\left|\nabla \vec{n}_{\vec{\xi}}\right|^{2} d x\right),
$$

where d satisfies

$$
\begin{aligned}
& \left|2 \pi d-\int_{\partial B_{r}} \partial_{r} \lambda d l_{\partial B_{r}}\right| \\
& \quad \leq C_{m}\left[\int_{B_{2 r}(0) \backslash B_{r}(0)}\left|\nabla \vec{n}_{\vec{\xi}}\right|^{2} d x+\frac{1}{\log R / r}\left(\|\nabla \lambda\|_{L^{2, \infty}(\Omega)}+\int_{\Omega}\left|\nabla \vec{n}_{\vec{\xi}}\right|^{2} d x\right)\right],
\end{aligned}
$$

and $C_{m}$ depends only on $m$, and $\lambda$ is as in lemma $\overline{V .2}$.

Proof of lemma V.3. It is convenient to introduce the constant

$$
\Lambda:=\|\nabla \lambda\|_{L^{2, \infty}(\Omega)}+\int_{\Omega}\left|\nabla \vec{n}_{\vec{\xi}}\right|^{2} d x
$$

We choose $\eta(m)$ smaller than the constant $\eta(m)$ given in lemma IV.3, so that there exists a framing

$$
\left\{\vec{e}_{1}, \vec{e}_{2}\right\} \in\left(W^{1,2}\left(\Omega, S^{m-1}\right)\right)^{2}
$$

with

$$
\star \vec{n}_{\vec{\xi}}=\vec{e}_{1} \wedge \vec{e}_{2} \quad \text { and } \quad \sum_{i=1}^{2} \int_{\Omega}\left|\nabla \vec{e}_{i}\right|^{2} d x \leq C \int_{\Omega}\left|\nabla \vec{n}_{\vec{\xi}}\right|^{2} d x
$$

The conformal parameter $\lambda$ satisfies

$$
\Delta \lambda=\nabla^{\perp} \vec{e}_{1} \cdot \nabla \vec{e}_{2} \quad \text { in } \Omega .
$$

Let $\mu$ be the solution of

$$
\begin{cases}\Delta \mu=\nabla^{\perp} \vec{e}_{1} \cdot \nabla \vec{e}_{2} & \text { in } \Omega \\ \mu=0 & \text { on } \partial \Omega .\end{cases}
$$

As in the proof of lemma V.2. Wente estimates give

$$
\|\mu\|_{L^{\infty}(\Omega)} \leq(2 \pi)^{-1}\left\|\nabla \vec{e}_{1}\right\|_{L^{2}}\left\|\nabla \vec{e}_{2}\right\|_{L^{2}} \leq C \Lambda
$$

and

$$
\|\nabla \mu\|_{L(\Omega)} \leq \sqrt{3 / 32 \pi}\left\|\nabla \vec{e}_{1}\right\|_{L^{2}}\left\|\nabla \vec{e}_{2}\right\|_{L^{2}} \leq C \Lambda .
$$

Let $\nu:=\lambda-\mu$ be the harmonic function in $\Omega$ equal to $\lambda$ on the boundary $\partial \Omega$. Analogously to (V.6) in the proof of lemma $\mathrm{V} .1$, there exists $\alpha \in(1 / 2,1)$ with

$$
\begin{aligned}
& \int_{\partial\left(B_{\alpha R} \backslash B_{\alpha^{-1} r}\right)}|\nabla \nu| \leq r\|\nabla \nu\|_{L^{1}\left(B_{2 r} \backslash B_{r}\right)}+2 R\|\nabla \nu\|_{L^{1}\left(B_{R} \backslash B_{R / 2}\right)} \\
& \leq C_{4}\|\nabla \nu\|_{L^{2, \infty}(\Omega)} \leq C_{4}\left[\|\nabla \lambda\|_{L^{2, \infty}(\Omega)}+\|\nabla \mu\|_{L(\Omega)}\right] \leq C_{4}(1+C) \Lambda
\end{aligned}
$$


where $C_{4}$ is a universal constant. From (V.36) we deduce the existence of two constants $a$ and $b$ depending on $\vec{\xi}$ such that

$$
\forall x \in \partial B_{\alpha^{-1} r}(0) \quad-C_{m} \Lambda \leq \nu(x)-a \leq C_{m} \Lambda \quad
$$

and

$$
\forall x \in \partial B_{\alpha R}(0) \quad-C_{m} \Lambda \leq \nu(x)-b \leq C_{m} \Lambda \quad,
$$

where $C_{m}>0$ only depends on $m$. Since the functions

$$
\tau^{ \pm}(x):=\frac{b-a}{\log R / r} \log \frac{|x|}{r}+a \pm C_{m} \Lambda
$$

are harmonic, and since (V.37) and (V.38) imply

$$
\tau^{-} \leq \nu \leq \tau^{+} \quad \text { on } \partial\left(B_{\alpha R} \backslash B_{\alpha^{-1} r}\right),
$$

the maximum principle yields

$$
\forall x \in B_{\alpha R} \backslash B_{\alpha^{-1} r} \quad-C_{m} \Lambda \leq \nu(x)-\left[\frac{b-a}{\log R / r} \log \frac{|x|}{r}+a\right] \leq C_{m} \Lambda
$$

Let $\rho \in(r, R)$, and define $2 \pi d:=\int_{\partial B_{\rho}} \partial_{\rho} \nu$. Integrating by parts gives

$$
\begin{aligned}
0 & =\int_{B_{\alpha R} \backslash B_{\alpha-1}} \Delta \nu \log |x| d x \\
& =2 \pi d \log \frac{R}{r}+\int_{0}^{2 \pi} \nu\left(\alpha^{-1} r, \theta\right) d \theta-\int_{0}^{2 \pi} \nu(\alpha R, \theta) d \theta .
\end{aligned}
$$

Hence

$$
\left|d-\frac{b-a}{\log R / r}\right| \leq \frac{C_{m} \Lambda}{\log R / r}
$$

Combining (V.39) and (V.40) yields

$$
\forall x \in B_{\alpha R} \backslash B_{\alpha^{-1} r} \quad-C_{m} \Lambda \leq \nu(x)-\frac{1}{2 \pi}\left[\int_{\partial B_{r}(0)} \partial_{r} \nu\right] \log \frac{|x|}{r}-a \leq C_{m} \Lambda
$$

We next estimate the difference $\int_{\partial B_{r}(0)} \partial_{r} \mu=\int_{\partial B_{r}(0)} \partial_{r}(\nu-\lambda)$. There holds

$$
\begin{aligned}
& 0=\int_{\Omega} \mu \Delta \log (|x| / R) d x=-\int_{\Omega} \nabla \mu \cdot \nabla \log (|x| / R) d x \\
& =-\log (r / R) \int_{\partial B_{r}} \partial_{r} \mu+\int_{\Omega}\left(\nabla^{\perp} \vec{e}_{1} \cdot \nabla \vec{e}_{2}\right) \log (|x| / R) d x
\end{aligned}
$$

As seen in the proof of lemmaIV.3, the construction of the moving frame $\left\{\vec{e}_{1}, \vec{e}_{2}\right\}$ requires to construct an extension $\hat{\vec{n}}$ of $\vec{n}_{\vec{\xi}}$ into $B_{r}(0)$. Since $\left\{\vec{e}_{1}, \vec{e}_{2}\right\}$ is also a moving frame for $\hat{\vec{n}}$ in $B_{R}(0)$, lemma IV.1 shows that

$$
\sum_{i=1}^{2} \int_{B_{R}(0)}\left|\nabla \vec{e}_{i}\right|^{2} d x \leq C \int_{\Omega}\left|\nabla \vec{n}_{\vec{\xi}}\right|^{2} d x \leq \Lambda
$$


Using this extension, we may also recast (V.42) in form

$$
\begin{aligned}
\log (R / r) & \int_{\partial B_{r}} \partial_{r} \mu=\int_{B_{R}}\left(\nabla^{\perp} \vec{e}_{1} \cdot \nabla \vec{e}_{2}\right) \log (|x| / R) d x \\
& -\int_{B_{r}}\left(\nabla^{\perp} \vec{e}_{1} \cdot \nabla \vec{e}_{2}\right) \log (|x| / R) d x .
\end{aligned}
$$

Let $\Psi$ be the solution of

$$
\left\{\begin{array}{l}
\Delta \Psi=\nabla^{\perp} \vec{e}_{1} \cdot \nabla \vec{e}_{2} \quad \text { in } B_{R}(0) \\
\Psi=0 \quad \text { on } \partial B_{R}(0)
\end{array}\right.
$$

As in the proof of lemma V.2. Wente estimates give

$$
\|\nabla \Psi\|_{L^{2,1}\left(B_{R}(0)\right)} \leq C_{0}\left\|\nabla \vec{e}_{1}\right\|_{L\left(B_{R}(0)\right)}\left\|\nabla \vec{e}_{2}\right\|_{L\left(B_{R}(0)\right)} \leq C_{m} \Lambda
$$

for some universal constant $C_{0}$ independent of $R$. Hence,

$$
\begin{gathered}
\left|\int_{B_{R}}\left(\nabla^{\perp} \vec{e}_{1} \cdot \nabla \vec{e}_{2}\right) \log (|x| / R) d x\right|=\left|\int_{B_{R}} \nabla \Psi \nabla \log (|x| / R) d x\right| \\
\leq\|\nabla \Psi\|_{L^{2,1}\left(B_{R}(0)\right)}\|\nabla \log (|x| / R)\|_{L^{2, \infty}\left(B_{R}(0)\right)} \leq C_{m} \Lambda
\end{gathered}
$$

On the other hand, lemma IV.3 and lemma IV.1 yield a frame

$$
\left\{\vec{f}_{1}, \vec{f}_{2}\right\} \in\left(W^{1,2}\left(B_{r}(0), S^{m-1}\right)\right)^{2}
$$

satisfying

$$
\star \hat{\vec{n}}=\overrightarrow{f_{1}} \wedge \overrightarrow{f_{2}}
$$

and

$$
\sum_{i=1}^{2} \int_{B_{r}(0)}\left|\nabla \vec{f}_{i}\right|^{2} d x \leq C \int_{B_{r}(0)}|\nabla \hat{\vec{n}}|^{2} d x \leq C_{m} \int_{B_{2 r}(0) \backslash B_{r}(0)}\left|\nabla \vec{n}_{\vec{\xi}}\right|^{2} d x \quad .
$$

Clearly, there exists $\theta \in W^{1,2}\left(B_{r}, \mathbb{R}\right)$ such that

$$
e^{i \theta}\left(\vec{e}_{1}+i \vec{e}_{2}\right)=\overrightarrow{f_{1}}+i \overrightarrow{f_{2}}
$$

and thus

$$
\vec{e}_{1} \cdot \nabla \vec{e}_{2}+\nabla \theta=\vec{f}_{1} \cdot \nabla \overrightarrow{f_{2}} \quad \text { in } B_{r}(0)
$$

Whence there holds

$$
\nabla^{\perp} \vec{e}_{1} \cdot \nabla \vec{e}_{2}=\nabla^{\perp} \vec{f}_{1} \cdot \nabla \overrightarrow{f_{2}} \quad \text { in } B_{r}(0)
$$

Let $\psi$ be the solution of

$$
\left\{\begin{array}{l}
\Delta \psi=\nabla^{\perp} \vec{f}_{1} \cdot \nabla \overrightarrow{f_{2}}-\left|B_{r}\right|^{-1} \int_{B_{r}} \nabla^{\perp} \vec{f}_{1} \cdot \nabla \overrightarrow{f_{2}} \\
\partial_{r} \psi=0 \quad \text { on } \partial B_{r}(0)
\end{array}\right.
$$

Calling again upon Wente estimates (this time with Neuman boundary data) gives the following control of the $L^{2,1}$-norm of $\nabla \psi$ :

$$
\|\nabla \psi\|_{L^{2,1}\left(B_{R}(0)\right)} \leq C_{0}\left\|\nabla \overrightarrow{f_{1}}\right\|_{L\left(B_{R}(0)\right)}\left\|\nabla \overrightarrow{f_{2}}\right\|_{L\left(B_{R}(0)\right)} \leq C_{m} \int_{B_{2 r}(0) \backslash B_{r}(0)}\left|\nabla \vec{n}_{\vec{\xi}}\right|^{2} d x
$$


Consequently, we derive

$$
\begin{aligned}
& \left|\int_{B_{r}}\left(\nabla^{\perp} \vec{e}_{1} \cdot \nabla \vec{e}_{2}\right) \log (|x| / R) d x\right| \leq \log (R / r)\left|\int_{B_{r}} \nabla^{\perp} \vec{f}_{1} \cdot \nabla \vec{f}_{2}\right|+\left|\int_{B_{r}} \nabla \psi \nabla \log (|x| / R) d x\right| \\
& \quad \leq C_{m} \log (R / r) \int_{B_{2 r}(0) \backslash B_{r}(0)}\left|\nabla \vec{n}_{\vec{\xi}}\right|^{2} d x+\|\nabla \psi\|_{L^{2,1}\left(B_{r}(0)\right)}\|\nabla \log (|x| / R)\|_{L^{2, \infty}\left(B_{R}(0)\right)} \\
& \quad \leq C_{m} \log (R / r) \int_{B_{2 r}(0) \backslash B_{r}(0)}\left|\nabla \vec{n}_{\vec{\xi}}\right|^{2} d x .
\end{aligned}
$$

Finally, combining (V.43), (V.45), and (V.49) gives the announced

$$
\log (R / r)\left|\int_{\partial B_{r}} \partial_{r} \mu\right| \leq C_{m}\left[\Lambda+\log (R / r) \int_{B_{2 r}(0) \backslash B_{r}(0)}\left|\nabla \vec{n}_{\vec{\xi}}\right|^{2} d x\right] .
$$

\section{Uniform $L^{2,1}$-control of the mean curvature vector of a Will- more immersion in neck regions}

The goal of this section is to prove the following result.

Lemma VI.1 Let $m \geq 3$. There exists a constant $\varepsilon(m)>0$ with the following property. Let $0<8 r<R$, and let $\vec{\xi}$ be any conformal Willmore immersion of $B_{R}(0)$ into $\mathbb{R}^{m}$ such that

$$
\sup _{r<s<R / 2} \int_{B_{2 s}(0) \backslash B_{s}(0)}\left|\nabla \vec{n}_{\vec{\xi}}\right|^{2} d x \leq \varepsilon(m) \quad .
$$

We set

$$
\Lambda:=\|\nabla \lambda\|_{L^{2, \infty}\left(B_{R} \backslash B_{r}\right)}+\int_{B_{R}(0)}\left|\nabla \vec{n}_{\vec{\xi}}\right|^{2} d x \quad,
$$

where $\lambda$ denotes as before the conformal parameter of $\vec{\xi}$. Then there holds

$$
\left\|e^{\lambda} \vec{H}_{\vec{\xi}}\right\|_{L^{2,1}\left(B_{R / 2} \backslash B_{2 r}\right)} \leq C(m, \Lambda)
$$

where $\vec{H}_{\vec{\xi}}$ is the mean curvature vector of the immersion $\vec{\xi}$, and $C(m, \Lambda)$ is a positive constant depending 10 only on $m$ and $\Lambda$.

Proof of lemma VI.1. According to the work in Ri2, an immersion $\vec{\xi}$ is conformal and Willmore on $B_{R}(0)$ if and only if there exists an $\mathbb{R}^{m}$-valued map $\vec{L}$ (uniquely defined up to an additive constant vector) satisfying

$$
\nabla \vec{L}=2 \nabla^{\perp} \vec{H}-3 \nabla^{\perp}\left(\pi_{\vec{n}}\right)(\vec{H})+\star\left(\nabla \vec{n}_{\vec{\xi}} \wedge \vec{H}\right)
$$

where $\star$ is the canonical Hodge operation on multivectors of $\mathbb{R}^{m}$ and $\pi_{\vec{n}}$ is the projection operator onto the normal space to $\vec{\xi}_{*} T \Sigma$. It is given by two consecutive applications of the contraction operator 11 with $\vec{n}$ (see (VI.87) in [Ri1]):

$$
\forall \vec{w} \in \mathbb{R}^{m} \quad \pi_{\vec{n}}(\vec{w}):=(-1)^{m-1} \vec{n}\llcorner(\vec{n}\llcorner\vec{w}) \quad .
$$

\footnotetext{
${ }^{10}$ the constant $C(m, \Lambda)$ is independent of the conformal type of the annulus.

${ }^{11}$ The contraction operator $L$ is the linear operation defined as follows. For every $\alpha, \beta$, and $\gamma$, respectively $p$, $q$, and $(p-q)$ vectors in $\mathbb{R}^{m}$, one has

$$
<\alpha\llcorner\beta, \gamma>=<\alpha, \beta \wedge \gamma>
$$
}


Hence the gradient of $\pi_{\vec{n}}$ in (VI.3) is to be understood as

$$
\forall \vec{w} \in \mathbb{R}^{m} \quad \nabla\left(\pi_{\vec{n}}\right)(\vec{w}):=(-1)^{m-1}(\nabla \vec{n})\left\llcorner(\vec{n} \mathbf{L} \vec{w})+(-1)^{m-1} \vec{n}\llcorner((\nabla \vec{n})\llcorner\vec{w}) \quad .\right.
$$

The first condition to impose on the constant $\varepsilon(m)$ is that it should be smaller than the one in lemma $\mathrm{V} .2$, so that the uniform Harnack estimate (V.14) holds on the annulus $B_{\alpha R} \backslash B_{\alpha^{-1} r}$ for every $0<\alpha<1$. If, in addition, we ensure that $\varepsilon(m)$ be smaller than the threshold constant appearing the $\epsilon$-regularity theorem I.5 in Ri2, then the uniform Harnack estimate enables 12 us to conclude that for any $x \in B_{4 R / 5}(0) \backslash B_{5 r / 4}(0)$ there holds

$$
\left|\nabla \vec{n}_{\vec{\xi}}(x)\right|^{2} \leq C|x|^{-2} \int_{B_{2|x|}(0) \backslash B_{|x| / 2}(0)}\left|\nabla \vec{n}_{\vec{\xi}}\right|^{2} d x \leq C \varepsilon(m) x^{-2},
$$

where $C$ is some constant independent of the data of the problem. This implies in particular that

$$
\left\|\nabla \vec{n}_{\vec{\xi}}\right\|_{L^{2, \infty}\left(B_{4 R / 5}(0) \backslash B_{5 r / 4}(0)\right)} \leq \sqrt{\frac{15 \pi}{16} C \varepsilon(m)}=C^{\prime} \sqrt{\varepsilon(m)} .
$$

Finally, we ask that $\varepsilon(m)>0$ satisfy $C^{\prime} \sqrt{\varepsilon(m)} \leq \sqrt{\eta(m)}$, where $\eta(m)$ is the constant given in lemmaV.3. Accordingly, there exist a constant $A$ (which depends a-priori on all parameters $r, R, \Lambda$, and $\vec{\xi}$ ) and a constant $d$ such that

$$
|d| \leq C_{m} \Lambda
$$

and

$$
\|\lambda(x)-d \log |x|-A\|_{L^{\infty}\left(B_{R / 2} \backslash B_{2 r}\right)} \leq C_{m} \Lambda
$$

We introduce for $0<s<R / 2$ the function

$$
\delta(s):=\left(s^{-2} \int_{B_{2 s}(0) \backslash B_{s / 2}(0)}\left|\nabla \vec{n}_{\vec{\xi}}\right|^{2} d x\right)^{1 / 2} .
$$

Clearly,

Inequality (VI.5) gives

$$
s \delta(s) \leq \sqrt{\Lambda} \quad \forall s \in(0, R / 2)
$$

$$
e^{\lambda(x)}\left|\vec{H}_{\vec{\xi}}(x)\right| \leq\left|\nabla \vec{n}_{\vec{\xi}}(x)\right| \leq C \delta(|x|) \quad \forall x \in B_{R / 2}(0) \backslash B_{2 r}(0)
$$

Clearly, since $8 r<R$, there holds

$$
\int_{r}^{R / 2} s \delta^{2}(s) d s \leq \log (4) \int_{B_{R}(0) \backslash B_{r / 2}(0)}\left|\nabla \vec{n}_{\vec{\xi}}\right|^{2} d x \leq 2 \Lambda .
$$

The identity (VI.3) shows that on any dyadic annulus $B_{2 s} \backslash B_{s}$ included in $B_{R}(0) \backslash B_{r}(0)$, the mean curvature vector satisfies

$$
\Delta \vec{H}=\frac{1}{2} \operatorname{div}\left(3 \nabla\left(\pi_{\vec{n}}\right)(\vec{H})+\star\left(\nabla^{\perp} \vec{n}_{\vec{\xi}} \wedge \vec{H}\right)\right)
$$

From (VI.10), a standard argument from the theory of second-order uniformly elliptic equations in divergence form then yields the existence of a constant $C_{1}>0$ such that

$$
e^{\lambda(x)}\left|\nabla \vec{H}_{\vec{\xi}}(x)\right| \leq C_{1} \frac{\delta(|x|)}{|x|} \quad \forall x \in B_{R / 2}(0) \backslash B_{2 r}(0) .
$$

\footnotetext{
12 indeed, the proof of theorem I.5 in [ $\mathrm{Ri2}$ requires that the conformal parameter $\lambda$ be "roughly" constant on balls around the origin. We are instead working on annuli, so the estimate V.14 is exactly what is needed.
} 
For any $t \in(r, R)$, we denote

$$
\vec{L}_{t}:=\frac{1}{\left|\partial B_{t}(0)\right|} \int_{\partial B_{t}(0)} \vec{L}
$$

where the function $\vec{L}$ is as (VI.10). Using (VI.3), (VI.8), and (VI.12), there holds

$$
\left|\vec{L}(x)-\vec{L}_{|x|}\right| \leq \int_{\partial B_{|x|}}|\nabla \vec{L}| \leq 2 \pi C_{1} e^{2 C_{m} \Lambda} e^{-\lambda(x)} \delta(|x|) \quad \forall x \in B_{R / 2}(0) \backslash B_{2 r}(0) \quad .
$$

This implies in particular that

$$
\int_{B_{R / 2}(0) \backslash B_{2 r}(0)} e^{2 \lambda(x)}\left|\vec{L}(x)-\vec{L}_{|x|}\right|^{2} d x \leq C_{2} \Lambda
$$

for some constant $C_{2}$. Note that we have used (VI.11).

We have next

$$
\frac{d \vec{L}_{t}}{d t}=\frac{1}{2 \pi} \int_{0}^{2 \pi} \frac{\partial \vec{L}}{\partial t}(t, \theta) d \theta=\frac{3}{2 \pi} \int_{0}^{2 \pi} \frac{1}{r} \frac{\partial \pi_{\vec{n}}}{\partial \theta}(\vec{H}) d \theta+\frac{1}{2 \pi} \int_{0}^{2 \pi} \star\left(\frac{\partial \vec{n}_{\vec{\xi}}}{\partial \nu} \wedge \vec{H}\right) d \theta
$$

Setting $a(t):=\left|\vec{L}_{t}\right|$, and putting (VI.10) into the latter gives the estimate

$$
|\dot{a}(t)|=\left|\frac{d\left|\vec{L}_{t}\right|}{d t}\right|=\left|\frac{d \vec{L}_{t}}{d t}\right| \leq C^{2} e^{-\lambda} \delta^{2}(t),
$$

Whence, using (VI.11), we reach

$$
\int_{2 r}^{R / 2} s e^{\lambda}|\dot{a}(s)| d s \leq 2 C^{2} \Lambda
$$

The Harnack estimate (VI.8) yields

$$
e^{A-C_{m} \Lambda}|x|^{d} \leq e^{\lambda(x)} \leq e^{A+C_{m} \Lambda}|x|^{d} \quad \forall x \in B_{R / 2} \backslash B_{2 r}(0)
$$

thereby showing that (VI.17) may be recast in the form

$$
\int_{2 r}^{R / 2} s^{1+d}|\dot{a}(s)| d s \leq 2 C^{2} e^{C_{m} \Lambda-A} \Lambda
$$

An elementary integration by parts gives for any $r \leq \tau<T \leq R$ :

$$
\int_{\tau}^{T} s^{1+d} \dot{a}(s) d s=T^{1+d} a(T)-\tau^{1+d} a(\tau)-(1+d) \int_{\tau}^{T} s^{d} a(s) d s
$$

Hence, since $a \geq 0$, we have

$$
\left\{\begin{array}{lll}
\forall d \leq-1 & \forall 2 r<t<R / 2 & t^{1+d} a(t) \leq(2 r)^{1+d} a(2 r)+\int_{2 r}^{R / 2} s^{1+d}|\dot{a}(s)| d s \\
\forall d \geq-1 & \forall 2 r<t<R / 2 & t^{1+d} a(t) \leq(R / 2)^{1+d} a(R / 2)+\int_{2 r}^{R / 2} s^{1+d}|\dot{a}(s)| d s
\end{array}\right.
$$


Recall that we still have the freedom to adjust the vector $\vec{L}$ by an additive constant vector. It will be convenient to us to choose $\vec{L}$ in such a way that

$$
\text { If } d \leq-1 \text { we take } \int_{\partial B_{2 r}} \vec{L}=0 \quad \text { whereas if } d \geq-1 \text { we take } \int_{\partial B_{R / 2}} \vec{L}=0 .
$$

This particular choice implies

$$
t e^{\lambda}\left|\vec{L}_{t}\right| \equiv t e^{\lambda} a(t) \leq 2 C^{2} e^{2 C_{m} \Lambda} \Lambda \quad \forall 2 r<t<R / 2 \quad,
$$

where we have used (VI.20), (VI.18) and (VI.19). With the help of an elementary computation, the latter then implies that

$$
\left\|e^{\lambda(x)}\left|\vec{L}_{|x|}\right|\right\|_{L^{2, \infty}\left(B_{R / 2} \backslash B_{2 r}\right)} \leq C_{0}(m, \Lambda),
$$

for some positive constant $C_{0}(m, \Lambda)$. Combining this inequality with (VI.14) gives

$$
\left\|e^{\lambda(x)} \vec{L}(x)\right\|_{L^{2, \infty}\left(B_{R / 2} \backslash B_{2 r}\right)} \leq C(m, \Lambda) \quad,
$$

where $C(m, \Lambda)$ depends only on $m$ and $\Lambda$. Moreover, the estimates (VI.13) and (VI.21) altogether yield

$$
e^{\lambda(x)}|\vec{L}(x)| \leq C\left(\Lambda, C_{m}\right)[1+|x| \delta(x)]|x|^{-1} \leq C^{\prime}(m, \Lambda)|x|^{-1} \quad \forall x \in B_{R / 2}(0) \backslash B_{2 r}(0) \quad .
$$

Note that we have also used (VI.9).

As is done in [Ri2] (see also section VI.7.2 of [Ri1]), we introduce on $B_{R}(0)$ the smooth function $S$ and the smooth $\bigwedge^{2}\left(\mathbb{R}^{m}\right)$-valued map $\vec{R}$ via

$$
\left\{\begin{array}{l}
\nabla S=\vec{L} \cdot \nabla \vec{\xi} \\
\nabla \vec{R}=\vec{L} \wedge \nabla \vec{\xi}+2\left(\star\left(\vec{n}_{\xi}\llcorner\vec{H})\right)\llcorner\nabla \vec{\xi} .\right.
\end{array}\right.
$$

Since $|\nabla \vec{\xi}|^{2}=2 e^{2 \lambda}$, the estimates (VI.6), (VI.23) give the bound

$$
\|\nabla S\|_{L^{2, \infty}\left(B_{R / 2} \backslash B_{2 r}\right)}+\|\nabla \vec{R}\|_{L^{2, \infty}\left(B_{R / 2} \backslash B_{2 r}\right)} \leq C(m, \Lambda) .
$$

Furthermore, using (VI.10), (VI.24), and (VI.9), we obtain the pointwise bound

$$
|\nabla S(x)|+|\nabla \vec{R}(x)| \leq C(m, \Lambda)|x|^{-1} \quad \forall x \in B_{R / 2} \backslash B_{2 r}
$$

One verifies (cf. Ri2] and theorem VI.15 of Ri1]) that the following equation 13 hold

$$
\left\{\begin{array}{l}
\nabla S=-(\star \vec{n}) \cdot \nabla^{\perp} \vec{R} \\
\nabla \vec{R}=(-1)^{m} \star\left(\vec{n} \bullet \nabla^{\perp} \vec{R}\right)+(\star \vec{n}) \nabla^{\perp} S .
\end{array}\right.
$$

\footnotetext{
${ }^{13}$ The linear operator $\bullet$ is the contraction which to a pair of $p$ and $q$-vectors of $\mathbb{R}^{m}$ assigns a $(p+q-2)$-vector of $\mathbb{R}^{m}$ in such a way that

and

$$
\forall \vec{a} \in \wedge^{p} \mathbb{R}^{m} \quad \forall \vec{b} \in \wedge^{1} \mathbb{R}^{m} \quad \vec{a} \bullet \vec{b}:=\vec{a}\llcorner\vec{b}
$$$$
\forall \vec{a} \in \wedge^{p} \mathbb{R}^{m} \quad \forall \vec{b} \in \wedge^{r} \mathbb{R}^{m} \quad \forall \vec{c} \in \wedge^{s} \mathbb{R}^{m}
$$$$
\vec{a} \bullet(\vec{b} \wedge \vec{c}):=(\vec{a} \bullet \vec{b}) \wedge \vec{c}+(-1)^{r s}(\vec{a} \bullet \vec{c}) \wedge \vec{b}
$$ 
For any $t \in(2 r, R / 2)$, we let

$$
S_{t}:=\frac{1}{\left|\partial B_{t}(0)\right|} \int_{\partial B_{t}(0)} S \quad, \quad \vec{R}_{t}:=\frac{1}{\left|\partial B_{t}(0)\right|} \int_{\partial B_{t}(0)} \vec{R} \quad \text { and } \quad \vec{n}_{t}:=\frac{1}{\left|\partial B_{t}(0)\right|} \int_{\partial B_{t}(0)} \vec{n} .
$$

The equations (VI.28) then yield

$$
\left\{\begin{aligned}
\frac{d S_{t}}{d t} & =\frac{1}{2 \pi} \int_{0}^{2 \pi} \frac{\partial S}{\partial t}(t, \theta) d \theta=\frac{1}{2 \pi} \int_{0}^{2 \pi} \star\left(\vec{n}-\vec{n}_{t}\right) \cdot \frac{1}{t} \frac{\partial \vec{R}}{\partial \theta} d \theta \\
\frac{d \vec{R}_{t}}{d t} & =\frac{1}{2 \pi} \int_{0}^{2 \pi} \frac{\partial \vec{R}}{\partial t}(t, \theta) d \theta \\
& =(-1)^{m-1} \frac{1}{2 \pi} \int_{0}^{2 \pi}\left(\vec{n}-\vec{n}_{t}\right) \bullet \frac{1}{t} \frac{\partial \vec{R}}{\partial \theta} d \theta-\frac{1}{2 \pi} \int_{0}^{2 \pi} \star\left(\vec{n}-\vec{n}_{t}\right) \frac{1}{t} \frac{\partial S}{\partial \theta} d \theta
\end{aligned}\right.
$$

Note that (VI.10) gives

$$
\left|\vec{n}(x)-\vec{n}_{|x|}\right| \leq C|x| \delta(|x|) \quad \forall x \in B_{R / 2}(0) \backslash B_{2 r}(0) \quad .
$$

Thus, (VI.27), (VI.29), and (VI.30) altogether yield

$$
\left|\frac{d S_{t}}{d t}\right|+\left|\frac{d \vec{R}_{t}}{d t}\right| \leq C(m, \Lambda) \delta(t) .
$$

Hence, with the help of (VI.11), we deduce

$$
\int_{2 r}^{R / 2}\left[\left|\frac{d S_{t}}{d t}\right|^{2}+\left|\frac{d \vec{R}_{t}}{d t}\right|^{2}\right] t d t \leq C(m, \Lambda)
$$

Our study requires the following result, proved in LaRi].

Lemma VI.2 LaRi] Let $a$ and $b$ be two functions on $B_{1}(0)$ such that $\nabla a \in L^{2, \infty}$ and $\nabla b \in L^{2}$. Let $0<\varepsilon<1 / 4$ and $\phi$ satisfy

$$
-\Delta \phi=\partial_{x_{1}} a \partial_{x_{2}} b-\partial_{x_{1}} b \partial_{x_{2}} a \quad \text { in } B_{1}(0) \backslash B_{\varepsilon}(0) \quad,
$$

For $\varepsilon \leq r \leq 1$, we set $\phi_{0}(r):=(2 \pi r)^{-1} \int_{\partial B_{r}(0)} \phi$, and we assume that

$$
\int_{\varepsilon}^{1}\left|\dot{\phi}_{0}\right|^{2} r d r<+\infty
$$

Then $\nabla \phi \in L^{2}\left(B_{1 / 2}(0) \backslash B_{2 \varepsilon}(0)\right)$ and there exists a positive constant $C$ independent of $\varepsilon$ and $\phi$ such that

$$
\begin{gathered}
\|\nabla \phi\|_{L^{2}\left(B_{1 / 2}(0) \backslash B_{2 \varepsilon}(0)\right)} \leq C\|\nabla a\|_{2, \infty}\|\nabla b\|_{2}+C\left\|\nabla \phi_{0}\right\|_{L^{2}\left(B_{1}(0) \backslash B_{\varepsilon}(0)\right)} \\
+C\|\nabla \phi\|_{L^{2, \infty}\left(B_{1}(0) \backslash B_{\varepsilon}(0)\right)}
\end{gathered}
$$


Applying divergence to both sides of the equations (VI.28) gives the conservative conformal Willmore system:

$$
\left\{\begin{aligned}
\Delta S & =-\star \nabla \vec{n} \cdot \nabla^{\perp} \vec{R} \\
\Delta \vec{R} & =(-1)^{m} \star\left(\nabla \vec{n} \bullet \nabla^{\perp} \vec{R}\right)+\star \nabla \vec{n} \cdot \nabla^{\perp} S .
\end{aligned}\right.
$$

Owing to (VI.1), (VI.26), and (VI.32), we may apply lemma VI.2 to the system (VI.35), and find

$$
\|\nabla S\|_{L^{2}\left(B_{R / 4}(0) \backslash B_{4 r}(0)\right)}+\|\nabla \vec{R}\|_{L^{2}\left(B_{R / 4}(0) \backslash B_{4 r}(0)\right)} \leq C(m, \Lambda) .
$$

Since the $L^{2}$-norm of $|x|^{-1}$ on annuli of the form $B_{a s}(0) \backslash B_{b s}(0)$ is independent of $s$, we can use the pointwise estimate (VI.27) to get an upper bound independent of $R$ and $r$ for

$$
\|\nabla S\|_{L^{2}\left(B_{R / 2}(0) \backslash B_{R / 4}(0) \cup B_{4 r}(0) \backslash B_{2 r}(0)\right)}+\|\nabla \vec{R}\|_{L^{2}\left(B_{R / 2}(0) \backslash B_{R / 4}(0) \cup B_{4 r}(0) \backslash B_{2 r}(0)\right)} .
$$

Combining it to (VI.36) then yields

$$
\|\nabla S\|_{L^{2}\left(B_{R / 2}(0) \backslash B_{2 r}(0)\right)}+\|\nabla \vec{R}\|_{L^{2}\left(B_{R / 2}(0) \backslash B_{2 r}(0)\right)} \leq C(m, \Lambda) .
$$

This new information, along with (VI.11), is injected into (VI.29) so as to produce

$$
\begin{aligned}
& \int_{2 r}^{R / 2}\left|\frac{d S_{t}}{d t}\right|+\left|\frac{d \vec{R}_{t}}{d t}\right| d t \leq C \int_{2 r}^{R / 2} \delta(t) \int_{\partial B_{t}}|\nabla S|+|\nabla \vec{R}| d t \\
& \quad \leq C\left(\int_{2 r}^{R / 2} \delta^{2}(t) t d t\right)^{1 / 2}\left(\int_{B_{R / 2} \backslash B_{2 r}}|\nabla S|^{2}+|\nabla \vec{R}|^{2} d x\right)^{1 / 2} \leq C(m, \Lambda) \quad .
\end{aligned}
$$

Since the functions $S$ and $\vec{R}$ are both defined up to an additive "constant", we have the freedom to impose the conditions $S_{2 r}=0$ and $\vec{R}_{2 r}=\overrightarrow{0}$. From (VI.31), we deduce in particular that

$$
\left|S_{t}\right|+\left|\vec{R}_{t}\right| \leq C(m, \Lambda) \quad \forall t \in(2 r, R / 2)
$$

Paired to the pointwise estimate (VI.27) on the gradient of $S$ and $\vec{R}$, the latter implies

$$
\|S\|_{L^{\infty}\left(B_{R / 2}(0) \backslash B_{2 r}(0)\right)}+\|\vec{R}\|_{L^{\infty}\left(B_{R / 2}(0) \backslash B_{2 r}(0)\right)} \leq C(m, \Lambda)
$$

We are sufficiently geared to apply the following general result, whose proof may be found in LaRi].

Lemma VI.3 LaRi] Let $a$ and $b$ be two functions on $B_{1}(0)$ such that $\nabla a \in L^{2}$ and $\nabla b \in L^{2}$. Let $0<\epsilon<1 / 4$ and $\phi$ satisfy

$$
-\Delta \phi=\partial_{x_{1}} a \partial_{x_{2}} b-\partial_{x_{1}} b \partial_{x_{2}} a \quad \text { in } B_{1}(0) \backslash B_{\varepsilon}(0) \quad .
$$

Assume that

$$
\|\phi\|_{\infty}<+\infty
$$

Then $\nabla \phi \in L^{2,1}\left(B_{1 / 2}(0) \backslash B_{2 \varepsilon}(0)\right)$, and there exists a positive constant $C$ independent of $\varepsilon$ and $\phi$ such that

$$
\begin{gathered}
\|\nabla \phi\|_{L^{2,1}\left(B_{1 / 2}(0) \backslash B_{2 \varepsilon}(0)\right)} \leq C\|\nabla a\|_{2}\|\nabla b\|_{2}+C\|\phi\|_{L^{\infty}\left(B_{1}(0) \backslash B_{\varepsilon}(0)\right)} \\
+C\|\nabla \phi\|_{L^{2}\left(B_{1}(0) \backslash B_{\varepsilon}(0)\right.} .
\end{gathered}
$$


Just as we did above, owing to (VI.1), (VI.26), and (VI.40), we apply lemma VI.3 to the system (VI.35), and find

$$
\|\nabla S\|_{L^{2,1}\left(B_{R / 4}(0) \backslash B_{4 r}(0)\right)}+\|\nabla \vec{R}\|_{L^{2,1}\left(B_{R / 4}(0) \backslash B_{4 r}(0)\right)} \leq C(m, \Lambda) \quad .
$$

Since the $L^{2,1}$-norm of $|x|^{-1}$ on annuli of the form $B_{a s}(0) \backslash B_{b s}(0)$ is independent of $s$, we use (VI.27) to get an upper bound independent of $R$ and $r$ for

$$
\|\nabla S\|_{L^{2,1}\left(B_{R / 2}(0) \backslash B_{R / 4}(0) \cup B_{4 r}(0) \backslash B_{2 r}(0)\right)}+\|\nabla \vec{R}\|_{L^{2,1}\left(B_{R / 2}(0) \backslash B_{R / 4}(0) \cup B_{4 r}(0) \backslash B_{2 r}(0)\right)} .
$$

Combining it to (VI.43) then yields

$$
\|\nabla S\|_{L^{2,1}\left(B_{R / 2}(0) \backslash B_{2 r}(0)\right)}+\|\nabla \vec{R}\|_{L^{2,1}\left(B_{R / 2}(0) \backslash B_{2 r}(0)\right)} \leq C(m, \Lambda) .
$$

It is shown in $\mathrm{Ri2}$ that

$$
4 e^{2 \lambda} \vec{H}_{\vec{\xi}} \equiv 2 \Delta \vec{\xi}=\nabla^{\perp} S \cdot \nabla \vec{\xi}-\nabla \vec{R}\left\llcorner\nabla^{\perp} \vec{\xi}\right.
$$

In particular, since $|\nabla \vec{\xi}|^{2}=2 e^{\lambda}$, calling upon (VI.44) in the latter yields the announced (VI.2), thereby completing the proof.

\section{VII $L^{2}$-weak energy quantization for the Gauss map of a Will- more immersion in neck regions}

Lemma VII.1 $\left[L^{2}\right.$-weak energy quantization for the Gauss map in neck regions] There exists a constant $\varepsilon(m)>0$ with the following property. Let $\vec{\xi}_{k}$ be a sequence of conformal Willmore immersions from $B_{R_{k}}(0)$ into $\mathbb{R}^{m}$, with $R_{k} \rightarrow+\infty$. Whenever $r_{k} \rightarrow 0$ satisfies

$$
\sup _{r \in\left(r_{k}, R_{k} / 2\right)} \int_{B_{2 r}(0) \backslash B_{r}(0)}\left|\nabla \vec{n}_{\vec{\xi}_{k}}\right|^{2} d x \leq \varepsilon(m),
$$

and 14

$$
\left\|\nabla \lambda_{k}\right\|_{L^{2, \infty}\left(\Omega_{k}\right)}+\int_{\Omega_{k}}\left|\nabla \vec{n}_{\vec{\xi}_{k}}\right|^{2} d x \leq \Lambda<+\infty \quad \forall k,
$$

where $\Lambda>0$ is independent of $k$ and $\Omega_{k}=B_{R_{k}}(0) \backslash B_{r_{k}}(0)$; then

$$
\forall \varepsilon \in(0, \varepsilon(m)) \quad \exists \alpha \in(0,1) \quad \text { s.t. } \quad \limsup _{k \rightarrow+\infty}\left\||x| \nabla \vec{n}_{\vec{\xi}_{k}}(x)\right\|_{L^{\infty}\left(B_{\alpha R_{k} / 2}(0) \backslash B_{\alpha^{-1} r_{k}}(0)\right)} \leq \varepsilon \text {. }
$$

Hence, in particular,

$$
\left.\forall \varepsilon \in(0, \varepsilon(m)) \quad \exists \alpha \in(0,1) \quad \text { s.t. } \quad \limsup _{k \rightarrow+\infty}\left\|\nabla \vec{n}_{\vec{\xi}_{k}}\right\|_{L^{2, \infty}\left(B_{\alpha R_{k} / 2}(0) \backslash B_{\alpha}-1 r_{k}\right.}(0)\right) \leq \varepsilon \quad .
$$

Proof of lemma VII.1. Analogously to the argument given in the paragraph following (VI.4) in the proof of lemma VI.1, the constant $\varepsilon(m)$ may be chosen so as to ensure that there holds

$$
\left|\nabla \vec{n}_{\vec{\xi}_{k}}(x)\right|^{2} \leq C|x|^{-2} \int_{B_{2|x|}(0) \backslash B_{|x| / 2}(0)}\left|\nabla \vec{n}_{\vec{\xi}_{k}}\right|^{2} d x \leq C \varepsilon(m) x^{-2} \quad \forall x \in B_{R_{k} / 2}(0) \backslash B_{2 r_{k}}(0),
$$

\footnotetext{
${ }^{14}$ as usual, $\lambda_{k}$ is the conformal parameter of $\vec{\xi}_{k}$.
} 
where $C$ is some constant independent of the data of the problem. This implies in particular that

$$
\left\|\nabla \vec{n}_{\vec{\xi}_{k}}\right\|_{L^{2, \infty}\left(B_{R_{k} / 2}(0) \backslash B_{2 r_{k}}(0)\right)} \leq C^{\prime} \sqrt{\varepsilon(m)} .
$$

We will argue by contradiction, by assuming that there exists a sequence of conformal Willmore immersions on $B_{R_{k}}(0)$ satisfying (VII.1) and (VII.2), but for which there exist $\varepsilon_{1}>0$ and $x_{k} \in \Omega_{k}$ with

$$
\log \left|\frac{\left|x_{k}\right|}{r_{k}}\right| \rightarrow+\infty \quad, \quad \log \left|\frac{\left|x_{k}\right|}{R_{k}}\right| \rightarrow-\infty \quad,
$$

and with

$$
\left|x_{k}\right|\left|\nabla \vec{n}_{\vec{\xi}_{k}}\left(x_{k}\right)\right| \geq \varepsilon_{1}>0
$$

From (VII.5), we deduce in particular that

$$
\int_{B_{2\left|x_{k}\right|}(0) \backslash B_{\left|x_{k}\right| / 2}(0)}\left|\nabla \vec{n}_{\vec{\xi}_{k}}\right|^{2} d x \geq \frac{\varepsilon_{1}^{2}}{C}>0
$$

We next demand that $\varepsilon(m)$ be smaller than its counterpart from lemma VI.1. Since all the conditions of this lemma are fulfilled, there exist $\vec{L}_{k} \in C^{\infty}\left(B_{R_{k}}(0), \mathbb{R}^{m}\right), S_{k} \in C^{\infty}\left(B_{R_{k}}(0), \mathbb{R}\right)$, and $\vec{R}_{k} \in$ $C^{\infty}\left(B_{R_{k}}(0), \bigwedge^{2} \mathbb{R}^{m}\right)$ such that

$$
\left\{\begin{aligned}
\nabla^{\perp} \vec{L}_{k} & :=\nabla \vec{H}_{k}-3 \pi_{\vec{n}_{\vec{\xi}_{k}}}\left(\nabla \vec{H}_{k}\right)+\star\left(\nabla^{\perp} \vec{n}_{\vec{\xi}_{k}} \wedge \vec{H}_{k}\right) \\
\nabla S_{k} & :=\vec{L}_{k} \cdot \nabla \vec{\xi}_{k} \\
\nabla \vec{R}_{k} & :=\vec{L}_{k} \wedge \nabla \vec{\xi}_{k}+2 \vec{H}_{k} \wedge \nabla^{\perp} \vec{\xi}_{k}
\end{aligned}\right.
$$

In the course of the proof of lemma VI.1, we have seen that

$$
\left\|e^{\lambda_{k}} \vec{L}_{\vec{\xi}_{k}}\right\|_{L^{2, \infty}\left(B_{R_{k} / 2} \backslash B_{2 r_{k}}\right)}+\left\|\nabla S_{k}\right\|_{L^{2,1}\left(B_{R_{k} / 2} \backslash B_{2 r_{k}}\right)}+\left\|\nabla \vec{R}_{k}\right\|_{L^{2,1}\left(B_{R_{k} / 2} \backslash B_{2 r_{k}}\right)} \leq C(m, \Lambda) .
$$

Moreover, we have the conservative conformal Willmore system

$$
\left\{\begin{array}{l}
\Delta S_{k}=-\left(\star \nabla \vec{n}_{\vec{\xi}_{k}}\right) \cdot \nabla^{\perp} \vec{R}_{k} \\
\Delta \vec{R}_{k}=(-1)^{m} \star\left(\nabla \vec{n}_{\vec{\xi}_{k}} \bullet \nabla^{\perp} \vec{R}_{k}\right)+\left(\star \nabla \vec{n}_{\vec{\xi}_{k}}\right) \cdot \nabla^{\perp} S_{k} \\
2 \Delta \vec{\xi}_{k}=\nabla^{\perp} S_{k} \cdot \nabla \vec{\xi}_{k}-\nabla \vec{R}_{k}\left\llcorner\nabla^{\perp} \vec{\xi}_{k} .\right.
\end{array}\right.
$$

Consider the conformal mapping

$$
\tilde{\vec{\xi}}_{k}(y):=e^{-\lambda_{k}\left(x_{k}\right)}\left(\vec{\xi}_{k}\left(\left|x_{k}\right| y\right)-\vec{\xi}_{k}\left(x_{k}\right)\right)
$$

An elementary computation shows that

$$
\nabla_{y} \vec{n}_{\tilde{\xi}_{k}}(y)=\left|x_{k}\right| \nabla_{x} \vec{n}_{\vec{\xi}_{k}}\left(\left|x_{k}\right| y\right),
$$

and

$$
\nabla_{y} \tilde{\vec{H}}_{k}(y)=\left|x_{k}\right| e^{-\lambda_{k}\left(x_{k}\right)} \nabla_{x} \vec{H}_{k}\left(\left|x_{k}\right| y\right),
$$

where $\tilde{\vec{H}}_{k}$ is the mean curvature vector of the immersion $\tilde{\vec{\xi}}_{k}$. The corresponding $\mathbb{R}^{m}$-valued map $\tilde{\vec{L}}_{k}$ satisfies

$$
\nabla_{y} \tilde{\vec{L}}_{k}(y)=\left|x_{k}\right| e^{-\lambda_{k}\left(x_{k}\right)} \nabla_{x} \vec{L}_{k}\left(\left|x_{k}\right| y\right)
$$


so that

$$
\tilde{\vec{L}}_{k}(y)=e^{-\lambda_{k}\left(x_{k}\right)} \vec{L}_{k}\left(\left|x_{k}\right| y\right)
$$

Clearly, the conformal factor of the rescaled immersion satisfies $\tilde{\lambda}_{k}(y)=\lambda_{k}\left(\left|x_{k}\right| y\right)-\lambda_{k}\left(x_{k}\right)$. This implies in particular that the corresponding $\tilde{S}_{k}$ and $\tilde{\vec{R}}_{k}$ are given by

$$
\tilde{S}_{k}(y)=S_{k}\left(\left|x_{k}\right| y\right) \quad \text { and } \quad \tilde{\vec{R}}_{k}(y)=\vec{R}_{k}\left(\left|x_{k}\right| y\right) \quad .
$$

We thus see that the maps $S_{k}$ and $\vec{R}_{k}$ behave under rescaling like the Gauss map $\vec{n}_{\vec{\xi}}$. This remarkable fact was first observed in [BR2], where it played a decisive role. From the latter and (VII.11), it follows that

$$
\limsup _{k \rightarrow+\infty}\left\|\nabla \tilde{S}_{k}\right\|_{L^{2,1}(K)}+\left\|\nabla \tilde{\vec{R}}_{k}\right\|_{L^{2,1}(K)} \leq C(m, \Lambda)
$$

for any compact subdomain $K$ of $\mathbb{C} \backslash\{0\}$.

The pointwise control of the conformal factor in neck regions provided by lemma $\nabla .3$ shows that the sequence of Willmore immersions $\tilde{\vec{\xi}}_{k}$ satisfies

$$
\limsup _{k \rightarrow+\infty}\left\|\nabla \vec{n}_{\tilde{\xi}_{k}}\right\|_{L_{l o c}^{\infty}(\mathbb{C} \backslash\{0\})}<+\infty
$$

and

$$
\limsup _{k \rightarrow+\infty}\left\|\log \left|\nabla \tilde{\vec{\xi}}_{k}\right|\right\|_{L_{l o c}^{\infty}(\mathbb{C} \backslash\{0\})}<+\infty
$$

From the work in $\left[\mathrm{Ri2}\right.$, we conclude that for all $l \in \mathbb{N}$, the sequence $\vec{\xi}_{k}$ converges in $C_{\text {loc }}^{l}(\mathbb{C} \backslash\{0\})$ to a Willmore immersion $\tilde{\vec{\xi}}_{\infty}$ of $\mathbb{C} \backslash\{0\}$. This strong convergence implies that the condition (VII.9) and the system (VII.12) pass to the limit. Hence,

$$
\int_{B_{2}(0) \backslash B_{1 / 2}(0)}\left|\nabla \vec{n}_{\tilde{\xi}_{\infty}}\right|^{2} d x \geq \frac{\varepsilon_{1}^{2}}{C}>0
$$

and

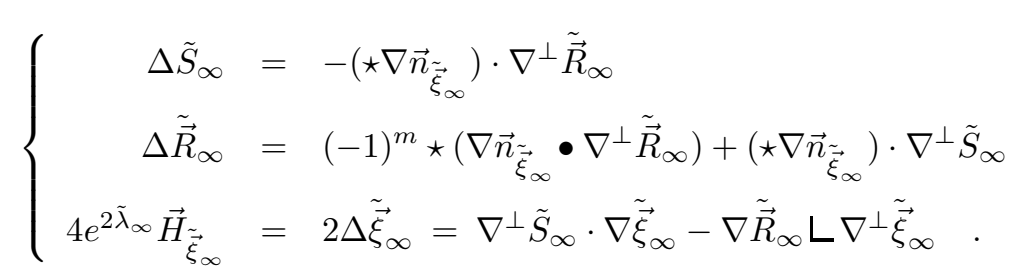

On one hand, we obtain from (VII.6) that

$$
\left\|\nabla \vec{n}_{\tilde{\xi}_{\infty}}\right\|_{L^{2, \infty}(\mathbb{C})} \leq C \sqrt{\varepsilon(m)} .
$$

On the other hand, it follows from (VII.13) that

$$
\left\|\nabla \tilde{S}_{\infty}\right\|_{L^{2,1}(\mathbb{C})}+\left\|\nabla \tilde{\vec{R}}_{\infty}\right\|_{L^{2,1}(\mathbb{C})} \leq C(m, \Lambda)<+\infty
$$

Applying to the system (VII.17) the Wente inequality from lemma IV.2 with $(p, q)=(2,1)$ yields now

$$
\begin{aligned}
\left\|\nabla \tilde{S}_{\infty}\right\|_{L^{2,1}(\mathbb{C})}+\left\|\nabla \tilde{\vec{R}}_{\infty}\right\|_{L^{2,1}(\mathbb{C})} & \leq C\left\|\nabla \vec{n}_{\tilde{\xi}_{\infty}}\right\|_{L^{2, \infty}(\mathbb{C})}\left[\left\|\nabla \tilde{S}_{\infty}\right\|_{L^{2,1}(\mathbb{C})}+\left\|\nabla \tilde{\vec{R}}_{\infty}\right\|_{L^{2,1}(\mathbb{C})}\right] \\
& \leq C_{m} \sqrt{\varepsilon(m)}\left[\left\|\nabla \tilde{S}_{\infty}\right\|_{L^{2,1}(\mathbb{C})}+\left\|\nabla \tilde{\vec{R}}_{\infty}\right\|_{L^{2,1}(\mathbb{C})}\right]
\end{aligned}
$$


where we have used (VII.18). Accordingly, when $C_{m} \sqrt{\varepsilon(m)}<1$, we deduce that $\nabla \tilde{S}_{\infty}=0$ and $\nabla \tilde{\vec{R}}_{\infty}=0$. Injecting this information in the last identity of (VII.17) gives

$$
\vec{H}_{\tilde{\xi}_{\infty}} \equiv \overrightarrow{0}
$$

In particular, since the (weighted) mean curvature vector is the trace part of the gradient of the Gauss map, it follows easily that

$$
\left|\nabla \vec{n}_{\tilde{\xi}_{\infty}}\right|^{2}=-2 e^{2 \tilde{\lambda}_{\infty}} K_{\tilde{\vec{\xi}}_{\infty}} \quad \text { on } \mathbb{C}
$$

where $K_{\tilde{\xi}_{\infty}}$ is the Gauss curvature of the limit immersion $\tilde{\vec{\xi}}_{\infty}$.

Let us next choose the constant $\varepsilon(m)$ small enough that we can apply lemma IV.3 with the condition (VII.18). Namely, on any ball $B_{\rho}(0)$, we construct a moving frame $\left\{\vec{e}_{1}^{\rho}, \vec{e}_{2}^{\rho}\right\}$ satisfying

$$
\star \hat{\vec{n}}_{\tilde{\xi}_{\infty}}=\vec{e}_{1}^{\rho} \wedge \vec{e}_{2}^{\rho} \quad, \quad \operatorname{div}\left(\vec{e}_{1}^{\rho} \cdot \nabla \vec{e}_{2}^{\rho}\right)=0
$$

and

$$
\int_{B_{R}(0)}\left|\nabla \vec{e}_{1}^{\rho}\right|^{2}+\left|\nabla \vec{e}_{2}^{\rho}\right|^{2} \leq C \int_{\mathbb{C}}\left|\nabla \vec{n}_{\tilde{\xi}_{\infty}}\right|^{2}
$$

We now consider a sequence of radii $\rho_{j}$ converging to infinity. Extracting a subsequence gives the existenceof a limiting frame $\left(\vec{e}_{1}^{\infty}, \vec{e}_{2}^{\infty}\right)$ on $\mathbb{C}$ satisfying

$$
\star \hat{\vec{n}}_{\tilde{\xi}_{\infty}}=\vec{e}_{1}^{\infty} \wedge \vec{e}_{2}^{\infty} \quad, \quad \operatorname{div}\left(\vec{e}_{1}^{\infty} \cdot \nabla \vec{e}_{2}^{\infty}\right)=0
$$

and

$$
\int_{\mathbb{C}}\left|\nabla \vec{e}_{1}^{\infty}\right|^{2}+\left|\nabla \vec{e}_{2}^{\infty}\right|^{2} \leq C \int_{\mathbb{C}}\left|\nabla \vec{n}_{\tilde{\xi}_{\infty}}\right|^{2}
$$

We have at every point of the minimal conformal immersion $\tilde{\vec{\xi}}_{\infty}$ the identity

$$
e^{2 \tilde{\lambda}_{\infty}} K_{\tilde{\vec{\xi}}_{\infty}}=-\nabla^{\perp} \vec{e}_{1}^{\infty} \cdot \nabla \vec{e}_{2}^{\infty}
$$

Integrating over $\mathbb{C}$ then yields

$$
\int_{\mathbb{C}} K_{\tilde{\xi}_{\infty}} e^{2 \tilde{\lambda}_{\infty}} d x=\int_{\mathbb{C}} d \vec{e}_{1}^{\infty} \dot{\wedge} d \vec{e}_{2}^{\infty}=0
$$

Paired to (VII.21), the latter implies that $\nabla \vec{n}_{\tilde{\xi}_{\infty}} \equiv 0$, which contradicts (VII.16). This is the desired contradiction, and thus lemma VII.1 is proved.

\section{Proof of theorems}

\section{VIII.1 Proof of theorem I.2}

Let $\vec{\Phi}_{k}$ be a sequence of Willmore immersions of $\Sigma$ with uniformly bounded energy

$$
\limsup _{k \rightarrow+\infty} E\left(\vec{\Phi}_{k}\right)<+\infty
$$

and such that the conformal class of the induced metric $g_{k}=\vec{\Phi}_{k}^{*} g_{\mathbb{R}^{m}}$ remains in a compact subdomain of the moduli space of $\Sigma$. 
We consider a subsequence, still denoted $\vec{\Phi}_{k}$, as given by lemma II.1. Let $\Xi_{k}$ be the corresponding sequence of Möbius transformations, and let $f_{k}$ be the sequence of Lipschitz diffeomorphisms of $\Sigma$, as given by lemma II.1. We set $\vec{\xi}_{k}:=\Xi_{k} \circ \vec{\Phi}_{k} \circ f_{k}$. The conclusion of lemma II.1 states that $\vec{\xi}_{k}$ weakly converges in $W^{2,2}$ to a weak immersion $\vec{\xi}_{\infty}$, away from finitely many points $a_{i}$. This limiting immersion might be branched at the points $a_{i}$.

Let $h_{k}$ be constant scalar curvature metric with respect to which $\vec{\xi}_{k}$ is conformal, and let $u_{k}$ be the function satisfying

$$
g_{k}=\vec{\xi}_{k}^{*} g_{\mathbb{R}^{m}}=e^{2 u_{k}} h_{k}
$$

The classical Liouville equation reads

$$
\Delta_{h_{k}} u_{k}+K_{g_{k}} e^{2 u_{k}}=K_{h_{k}}
$$

Since $h_{k}$ strongly converges to some limiting constant scalar curvature $h_{\infty}$, classical results from geometric analysis on manifolds (see for instance $\mathrm{Aub}$ ) show that the gradient of the Green function associated with the Laplace-Beltrami operator $\Delta_{h_{k}}$ is uniformly bounded in $L^{2, \infty}$. Hence,

$$
\left\|\nabla u_{k}\right\|_{L^{2, \infty}(\Sigma)} \leq C\left[\int_{\Sigma}\left|K_{g_{k}}\right| \operatorname{dvol}_{g_{k}}+\int_{\Sigma}\left|K_{h_{k}}\right| d v o l_{h_{k}}\right] \leq C\left[E\left(\vec{\Phi}_{k}\right)+2 \pi|\chi(\Sigma)|\right]
$$

where the $L^{2, \infty}$-norm is to be understood with respect to a reference metric $g_{0}$ on $\Sigma$. As before, $\chi(\Sigma)$ denotes the Euler characteristic of $\Sigma$. We define the constant

$$
\Lambda:=\sup _{k \in \mathbb{N}} E\left(\vec{\Phi}_{k}\right)+\left\|\nabla u_{k}\right\|_{L^{2, \infty}(\Sigma)}<+\infty
$$

Owing to the $\varepsilon$-regularity theorem I.5 in Ri2, the sequence $\vec{\xi}_{k}$ actually converges strongly in $C_{l o c}^{l}(\Sigma \backslash$ $\left.\left\{a_{1}, \ldots, a_{N}\right\}, \mathbb{R}^{m}\right)$, for all $l \in \mathbb{N}$. Namely,

$$
\vec{\xi}_{k} \longrightarrow \vec{\xi}_{\infty} \quad \text { in } \quad C_{l o c}^{l}\left(\Sigma \backslash\left\{a_{1}, \ldots, a_{N}\right\}, \mathbb{R}^{m}\right)
$$

Accordingly, the limiting immersion $\vec{\xi}_{\infty}$ is Willmore, although a priori only away from the points $\left\{a_{1}, \ldots, a_{N}\right\}$. Since $\vec{\xi}_{k}$ is Willmore, for any fixed small radius $\rho>0$ and any $i=1, \ldots, N$, one has

$$
\int_{B_{\rho}\left(a_{i}\right)} \operatorname{div}\left(2 \nabla \vec{H}_{\vec{\xi}_{k}}-3 \nabla\left(\pi_{\vec{n}_{\vec{\xi}_{k}}}\right)\left(\vec{H}_{\vec{\xi}_{k}}\right)-\star\left(\nabla^{\perp} \vec{n}_{\vec{\xi}_{k}} \wedge \vec{H}_{\vec{\xi}_{k}}\right)\right) d x=0
$$

where $B_{\rho}\left(a_{i}\right)$ is the geodesic ball centered at $a_{i}$ and of radius $\rho$ for the flat metric, in some converging conformal coordinate system for $\vec{\xi}_{k}^{*} g_{\mathbb{R}^{m}}$. Whence,

$$
\left.\int_{\partial B_{\rho}\left(a_{i}\right)} 2 \partial_{\nu} \vec{H}_{\vec{\xi}_{k}}-3 \partial_{\nu}\left(\pi_{\vec{n}_{\vec{\xi}_{k}}}\right)\left(\vec{H}_{\vec{\xi}_{k}}\right)+\star\left(\partial_{\tau} \vec{n}_{\vec{\xi}_{k}} \wedge \vec{H}_{\vec{\xi}_{k}}\right)\right) \rho d \theta=0
$$

where $\nu$ is the unit normal vector $x /|x|$ and $\tau$ the unit tangent vector $x^{\perp} /|x|$ to the boundary of $B_{\rho}\left(a_{i}\right)$. The strong convergence of $\vec{\xi}$ to $\vec{\xi}_{\infty}$ enables passing to the limit in the last identity, and we thus find

$$
\left.\int_{\partial B_{\rho}\left(a_{i}\right)} 2 \partial_{\nu} \vec{H}_{\vec{\xi}_{\infty}}-3 \partial_{\nu}\left(\pi_{\vec{n}_{\vec{\xi}_{\infty}}}\right)\left(\vec{H}_{\vec{\xi}_{\infty}}\right)+\star\left(\partial_{\tau} \vec{n}_{\vec{\xi}_{\infty}} \wedge \vec{H}_{\vec{\xi}_{\infty}}\right)\right) \rho d \theta=0
$$

for all fixed $\rho$.

This is precisely the condition required to apply the "point removability" theorem established in Ri2]. 
In particular, $\vec{\xi}_{\infty}$ extends to a (possibly branched), smooth Willmore immersion in $B_{\rho}\left(a_{i}\right)$. More detailed information on the behavior of the immersion near the points $a_{i}$ are given in BR2.

We now choose a fixed $\varepsilon_{0}>0$ smaller than the constants $\varepsilon(m)$ of lemmas VI.1 and VII.1 For this particular choice of $\varepsilon_{0}$, we apply the bubble-neck decomposition procedure outlined in proposition III.1. Owing to (III.13), both lemma VI.1 and lemma VII.1 apply in each connected component of $\Omega_{k}(\alpha)$, which, recall, is a union of disjoint annuli. From lemma VI.1, we thus have

$$
\limsup _{k \rightarrow+\infty}\left\|e^{\lambda} \vec{H}_{\vec{\xi}_{k}}\right\|_{L^{2,1}\left(\Omega_{k}(1 / 2)\right)} \leq C(m, \Lambda) .
$$

As for lemma VII.1 it yields

$$
\forall \varepsilon>0 \quad \exists \alpha>0 \quad \text { s.t. } \quad\left\|\nabla \vec{n}_{\vec{\xi}_{k}}\right\|_{L^{2, \infty}\left(\Omega_{k}(\alpha)\right) \leq \varepsilon .} .
$$

Combining this last assertion with the result of lemma V.1 implies that

$$
\forall \varepsilon>0 \quad \exists \alpha>0 \quad \text { s.t. } \quad\left|\int_{\Omega_{k}(\alpha)} K_{\vec{\xi}_{k}} d v o l_{g_{k}}\right| \leq \varepsilon .
$$

Altogether now, (VIII.4 and (VIII.5) give

$$
\begin{aligned}
& \forall \varepsilon>0 \quad \exists \alpha>0 \quad \exists k_{0} \in \mathbb{N} \quad \text { s.t. } \quad \forall k \geq k_{0} \\
& \left\|e^{\lambda} \vec{H}_{\vec{\xi}_{k}}\right\|_{L^{1}\left(\Omega_{k}(\alpha)\right)} \leq\left\|e^{\lambda} \vec{H}_{\vec{\xi}_{k}}\right\|_{L^{2,1}\left(\Omega_{k}(\alpha)\right)}^{1 / 2}\left\|e^{\lambda} \vec{H}_{\vec{\xi}_{k}}\right\|_{L^{2, \infty}\left(\Omega_{k}(\alpha)\right)}^{1 / 2} \leq \sqrt{C(m, \Lambda) \varepsilon},
\end{aligned}
$$

where we used the fact that $L^{2, \infty}$ is the dual space of $L^{2,1}$.

Combining (VIII.7) and (VIII.6) shows that

$$
\lim _{\alpha \rightarrow 0} \lim _{k \rightarrow+\infty} \int_{\Omega_{k}(\alpha)}\left|\nabla \vec{n}_{\vec{\xi}_{k}}\right|^{2} d x=0 .
$$

This is the no-neck energy property which we aimed at establishing. To finish the proof of theorem I.2. there remains to identify the energy of the bubbles as converging in the limit to that of Willmore spheres. This is what we do below.

Let $i \in\{1, \ldots, N\}$ and let $j \in\left\{1, \ldots, Q^{i}\right\}$. From (III.15), on the bubble domain

$$
B(i, j, \alpha, k):=B_{\alpha^{-1}} \rho_{k}^{i, j}\left(x_{k}^{i, j}\right) \backslash \cup_{j^{\prime} \in I^{i, j}} B_{\alpha \rho_{k}^{i, j}}\left(x_{k}^{i, j^{\prime}}\right)
$$

there is no concentration of energy. Hence, arguing as in the proof of lemma V.2 (see also lemma III.1 in [Ri3]), the conformal factor satisfies a uniform Harnack estimate:

$$
\forall 0<\alpha<1 \quad \exists C_{\alpha}>0 \quad \text { s.t. } \quad 1 \leq \frac{\sup _{\{x \in B(i, j, \alpha, k)\}} e^{\lambda_{k}}(x)}{\inf _{\{x \in B(i, j, \alpha, k)\}} e^{\lambda_{k}}(x)} \leq C_{\alpha}
$$

Choose an arbitrary point

$$
z_{k}^{i, j} \in B(i, j, \alpha, k) \cap B_{\rho_{k}^{i, j}}\left(x_{k}^{i, j}\right) \backslash\left(\cup_{j^{\prime} \in I^{i, j}} B_{M^{-1} \rho_{k}^{i, j}}\left(x_{k}^{i, j^{\prime}}\right)\right),
$$

where $M>\operatorname{card}\left(I^{i, j}\right)$; and set $\lambda(i, j, k, \alpha):=\lambda_{k}\left(x_{k}\right)$. We introduce the renormalized Willmore immersion

$$
\tilde{\vec{\xi}}_{k}(y):=e^{-\lambda(i, j, \alpha, k)}\left[\vec{\xi}_{k}\left(\rho_{k}^{i, j} y+x_{k}^{i, j}\right)-\vec{\xi}_{k}\left(z_{k}^{i, j}\right)\right] .
$$


We can extract a subsequence in such a way that the following limits exist:

$$
\forall j^{\prime} \in I^{i, j} \quad \lim _{k \rightarrow+\infty} \frac{x_{k}^{i, j}-z_{k}^{i, j}}{\rho_{k}^{i, j}}=a_{i}^{j, j^{\prime}} \in B_{1}(0) \backslash\{0\} .
$$

As the energy does not concentrate on any $B(i, j, \alpha, k)$ for $0<\alpha<1$, we obtain, using once more the $\varepsilon$-regularity and the local control of the conformal factor ensured by (VIII.9), that

$$
\tilde{\vec{\xi}}_{k} \longrightarrow \tilde{\vec{\xi}}_{\infty} \quad \text { in } \quad C_{l o c}^{l}\left(\mathbb{C} \backslash \cup_{j^{\prime} \in I^{i, j}}\left\{a_{i}^{j, j^{\prime}}\right\}\right) \quad \forall l \in \mathbb{N}
$$

where $\tilde{\vec{\xi}}_{\infty}$ is a Willmore immersion of $\mathbb{C} \backslash \cup_{j^{\prime} \in I^{i, j}}\left\{a_{i}^{j, j^{\prime}}\right\}$. Suppose that

$$
\int_{\mathbb{C}}\left|\nabla \tilde{\vec{\xi}}_{\infty}\right|^{2} d x<+\infty
$$

Then, using the stereographic projection of $S^{2}$ onto $\mathbb{C}$ with respect to the north pole, $\tilde{\vec{\xi}}_{\infty}$ realizes an immersion of $S^{2} \backslash \cup_{j^{\prime} \in I^{i, j}}\left\{a_{i}^{j, j^{\prime}}\right\} \cup\{N o r t h\}$ included in a bounded domain. Just as we explained above for the limit immersion $\vec{\xi}_{\infty}$, one can remove the singularities $\cup_{j^{\prime} \in I^{i, j}}\left\{a_{i}^{j, j^{\prime}}\right\} \cup\{N o r t h\}$, which possibly realize branch points of our Willmore $S^{2}$. We have moreover

$$
\lim _{\alpha \rightarrow 0} \lim _{k \rightarrow+\infty} \int_{B(i, j, \alpha, k)} e^{2 \lambda_{k}}\left|\vec{H}_{\vec{\xi}_{k}}\right|^{2}=W\left(\tilde{\vec{\xi}}_{\infty}\right)
$$

On the other hand, suppose that 15

$$
\int_{\mathbb{C}}\left|\nabla \tilde{\vec{\xi}}_{\infty}\right|^{2} d x_{1} d x_{2}=+\infty
$$

Following $\mathrm{Ri4}$, we can find a point $p_{k} \in \mathbb{R}$ such that

$$
\forall k \in \mathbb{N} \quad e^{-\lambda(i, j, \alpha, k)}\left[\vec{\xi}_{k}(\Sigma)-\vec{\xi}_{k}\left(z_{k}^{i, j}\right)\right] \cap B_{1}\left(p_{k}\right)=\emptyset \quad \text { and } \quad \limsup _{k \rightarrow+\infty}\left|p_{k}\right|<+\infty \quad .
$$

Let $\mathcal{I}_{k}$ be the inversion with respect to $p_{k}$, and $\hat{\vec{\xi}}_{k}:=\mathcal{I}_{k} \circ \tilde{\vec{\xi}}_{k}$. We may assume, modulo extraction of a subsequence if necessary, that the sequence $p_{k}$ converges to a point $p_{\infty}$. We let $\mathcal{I}_{\infty}$ be the inversion in $\mathbb{R}^{m}$ with respect to this limit-point. There holds

$$
\hat{\vec{\xi}}_{k} \longrightarrow \hat{\vec{\xi}}_{\infty}=\mathcal{I}_{\infty} \circ \tilde{\vec{\xi}}_{\infty} \quad \text { in } \quad C_{l o c}^{l}\left(\mathbb{C} \backslash \cup_{j^{\prime} \in I^{i, j}}\left\{a_{i}^{j, j^{\prime}}\right\}\right) \quad \forall l \in \mathbb{N}
$$

where $\hat{\vec{\xi}}_{\infty}$ is a Willmore immersion of $\mathbb{C} \backslash \cup_{j^{\prime} \in I^{i, j}}\left\{a_{i}^{j, j^{\prime}}\right\}$ satisfying

$$
\int_{\mathbb{C}}\left|\nabla \hat{\vec{\xi}}_{\infty}\right|^{2} d x<+\infty
$$

Arguing as above, $\hat{\vec{\xi}}_{\infty}$ extends to a smooth, possibly branched, immersion of $S^{2}$. Using lemma A.4 in [Ri4], we then obtain that

$$
\lim _{\alpha \rightarrow 0} \lim _{k \rightarrow+\infty} \int_{B(i, j, \alpha, k)} e^{2 \lambda_{k}}\left|\vec{H}_{\vec{\xi}_{k}}\right|^{2} d x=W\left(\hat{\vec{\xi}}_{\infty}\right)-4 \pi \theta_{0}
$$

\footnotetext{
${ }^{15}$ This should correspond to the case when $\lambda(i, j, \alpha, k) \rightarrow-\infty$.
} 
where $\theta_{0}$ is the integer density of $\vec{\xi}_{\infty}(\mathbb{C})$ at the image point $0 \in \mathbb{R}^{m}$.

Altogether, (VIII.3), (VIII.9), and (VIII.11), imply that there exists a subsequence (indexed as the original, for notational convenience) such that

$$
\lim _{k \rightarrow+\infty} W\left(\vec{\Phi}_{k}\right)=W\left(\vec{\xi}_{\infty}\right)+\sum_{s=1}^{p} W\left(\tilde{\vec{\xi}}_{\infty}^{s}\right)+\sum_{t=1}^{q}\left[W\left(\hat{\vec{\xi}}_{\infty}\right)-4 \pi \theta_{0}^{t}\right]
$$

The index $s$ is used for the first alternative, in which the bubble has no infinite end ; while the index $t$ is used for the second alternative, in which the bubble has at least one infinite planar end, and it must thus be inverted to become compact. This is exactly the desired energy identity (I.2), thereby ending the proof of theorem I.2.

\section{VIII.2 Proof of theorem I.5}

Let $\vec{\Phi}_{k}$ be a sequence of Willmore immersions satisfying

$$
W\left(\vec{\Phi}_{k}\right)<\min \left\{8 \pi, \omega_{g}^{m}\right\}-\delta \quad .
$$

We want to show that it is possible extract a subsequence $\vec{\Phi}_{k^{\prime}}$, and to find a sequence of diffeomorphisms $f_{k^{\prime}}$ of $\Sigma$ and Moebius transformationa $\Xi_{k^{\prime}}$, such that

$$
\vec{\xi}_{k^{\prime}}:=\Xi_{k^{\prime}} \circ \vec{\Phi}_{k^{\prime}} \circ f_{k^{\prime}} \longrightarrow \vec{\xi}_{\infty} \quad \text { strongly in } C^{l}(\Sigma) \quad \forall l \in \mathbb{N}
$$

From the work in Ri4] (cf. also [KuLi]), the sequence of conformal classes of the metrics induced by $\vec{\Phi}_{k}$ remains in a compact subdomain of the Moduli space. Theorems $I .3$ and I.4 whence apply. If

$$
\lim _{k^{\prime} \rightarrow \infty} W\left(\vec{\Phi}_{k^{\prime}}\right)=W\left(\vec{\xi}_{\infty}\right)
$$

then from theorem [.3. we get (VIII.13) directly. If not, then there must be a bubble. We take it to be the most concentrated one, centered at $x_{k^{\prime}}$ and of radius $\rho_{k^{\prime}}$, and converging to a point $a^{1}$. As is explained at the end of the previous section, we can find a Moebius transformation $\Lambda_{k^{\prime}}$ such that in local conformal coordinates there holds

$$
\Lambda_{k^{\prime}} \circ \vec{\xi}_{k^{\prime}}\left(\rho_{k^{\prime}} y+x_{k^{\prime}}\right) \longrightarrow \hat{\vec{\xi}}_{\infty}
$$

where $\hat{\vec{\xi}}_{\infty}$ is a Willmore sphere. Assume this sphere is homothetic to the round sphere. Then, if $\Lambda_{k^{\prime}}$ contains no inversion and $\hat{\vec{\xi}}_{\infty}$ is of the type $\eta_{s}$ from theorem [.2. we conclude that

$$
\lim _{k^{\prime} \rightarrow \infty} W\left(\vec{\Phi}_{k^{\prime}}\right) \geq W\left(\vec{\xi}_{\infty}\right)+W\left(\hat{\vec{\xi}}_{\infty}\right) \geq 4 \pi+4 \pi=8 \pi \quad
$$

which is a contradiction.

If now $\Lambda_{k^{\prime}}$ contains an inversion, then modulo a dilation in the image, we can arrange for $\xi_{k^{\prime}}\left(\rho_{k^{\prime}} y+x_{k^{\prime}}\right)$ to converge strongly to a plane, and thus

$$
\int_{B_{\rho_{k^{\prime}}}\left(x_{k^{\prime}}\right)}\left|\nabla \vec{\xi}_{k^{\prime}}\right| \rightarrow 0
$$

This contradicts the very definition of a bubble, which requires that

$$
\int_{B_{\rho_{k^{\prime}}}\left(x_{k^{\prime}}\right)}\left|\nabla \vec{\xi}_{k^{\prime}}\right| \geq \varepsilon(m)>0
$$


Accordingly, $\hat{\vec{\xi}}_{\infty}$ can not be homothetic to the round sphere. Calling upon the results from Bry and Mon, it follows that

$$
\lim _{k^{\prime} \rightarrow \infty} W\left(\vec{\Phi}_{k^{\prime}}\right)=\lim _{k^{\prime} \rightarrow \infty} W\left(\Lambda_{k^{\prime}} \circ \vec{\xi}_{k^{\prime}}\right) \geq \lim _{k^{\prime} \rightarrow \infty} W\left(\Lambda_{k^{\prime}} \circ \xi_{k^{\prime}}\left(\rho_{k^{\prime}} y+x_{k^{\prime}}\right)\right) \longrightarrow W\left(\hat{\vec{\xi}}_{\infty}\right) \geq 8 \pi,
$$

which is again a contradiction.

Therefore, we have shown that in all cases, the assertion (VIII.13) holds, thereby proving theorem I.5.

\section{References}

[Aub] Aubin, Thierry "Some non-linear problems in riemannian geometries" Springer (1998)

[BK] Bauer, Matthias; Kuwert, Ernst "Existence of minimizing Willmore surfaces of prescribed genus." Int. Math. Res. Not. (2003), no. 10, 553-576.

[BR1] Bernard, Yann; Rivière, Tristan "Local Palais Smale Sequences for the Willmore Functional" arXiv:0904.0360v1 (2009).

[BR2] Bernard, Yann; Rivière, Tristan "Asymptotic Analysis of Branched Willmore Surfaces" preprint (2011).

[BR3] Bernard, Yann; Rivière, Tristan "Loss of energy of Willmore immersions with conformal classes degenerating in Moduli space", to appear.

[Bla3] Blaschke, Wilhelm "Vorlesungen über Differentialgeometrie und geometrische grundlagen von Einsteins relativitätstheorie" III Die Grundlehren der mathematischen wissenschaften in einzeldarstellungen. Bd. XXIX Differentialgeometrie der Kreise und Kugeln, bearbeitet von Gerhard Thomsen. 1929

[Bry] Bryant, Robert L. "A duality theorem for Willmore surfaces." J. Diff. Geom. 20 (1984), no. 1, $23-53$.

[BuPa] Butscher, Adrian; Pacard, Frank Doubling constant mean curvature tori in S3. Ann. Sc. Norm. Super. Pisa Cl. Sci. (5) 5 (2006), no. 4, 611638.

[DiT] W. Ding and G. Tian "Energy identity for a class of approximate harmonic maps from surfaces" Comm. Anal. Geom. 3 (1995), no.3-4, 543-554.

[Ge] Ge, Yuxin "A remark on generalized harmonic maps into spheres." Nonlinear Anal. 36 (1999), no. 4, Ser. A: Theory Methods, 495506.

[GT] Gilbarg, David; Trudinger, Neil S. "Elliptic partial differential equations of second order". Reprint of the 1998 edition. Classics in Mathematics. Springer-Verlag, Berlin, 2001.

[Hel] Hélein, Frédéric "Harmonic Maps, Conservation Laws, and Moving Frames." Cambridge Tracts in Mathematics, 150. Cambridge University Press (2002).

[Jo] J. Jost, "Two-dimensional geometric variational problems", Wiley, (1991).

[KuLi] Kuwert, Ernst; Yuxiang Li " $W^{2,2}$-conformal immersions of a closed Riemann surface into $\mathbb{R}^{n}$ " arXiv:1007.3967 (2010).

[KS] Kuwert, Ernst; Schätzle, Reiner "Removability of point singularities of Willmore surfaces". Ann. of Math. (2) 160 (2004), no. 1, 315-357. 
[KS1] Kuwert, Ernst; Schätzle, Reiner Closed surfaces with bounds on their Willmore energy , Preprint Centro di Ricerca Matematica Ennio De Giorgi, Pisa, 2008.

[LaRi] Laurain, Paul; Rivière, Tristan "Lorentz-Wente uniform estimates on degenerating annuli" preprint (2011).

[LiRi1] Lin, Fang-Hua; Rivière, Tristan A quantization property for moving line vortices. Comm. Pure Appl. Math. 54 (2001), no. 7, 826850.

[LiRi2] Lin, Fang-Hua; Rivière, Tristan Energy quantization for harmonic maps. Duke Math. J. 111 (2002), no. 1, 177193.

[Mon] Montiel, Sebastiàn "Willmore two-spheres in the four-sphere." Trans. Amer. Math. Soc. 352 (2000), no. 10, 4469-4486.

[MS] Müller, Stefan; Šverák, Vladimír “On surfaces of finite total curvature.” J. Diff. Geom. 42 (1995), no. 2, 229-258.

[Pa] T.Parker, "Bubble tree convergence for harmonic maps", J. Diff. Geom. 44 (1996), 545-633.

[Ri1] Rivière, Tristan "Conformally Invariant 2-dimensional Variational Problems" Cours joint de l'Institut Henri Poincaré - Paris XII Creteil, Novembre 2010.

[Ri2] Rivière, Tristan "Analysis aspects of Willmore surfaces", Inventiones Math., 174 (2008), no.1, 1-45.

[Ri3] Rivière, Tristan "Variational Principles for immersed Surfaces with $L^{2}$-bounded Second Fundamental Form." arXiv:1007.2997 july 2010.

[Ri4] Rivière, Tristan "Lipschitz conformal immersions from degenerating Riemann surfaces with $L^{2}-$ bounded second fundamental forms." preprint (2010).

[Ri5] Rivière, Tristan Interpolation spaces and energy quantization for Yang-Mills fields. Comm. Anal. Geom. 10 (2002), no. 4, 683708.

[Ri6] Rivière, Tristan "Bubbling and regularity issues in geometric non-linear analysis" Proceedings of the International Congress of Mathematicians, Vol. III (Beijing, 2002), 197208, Higher Ed. Press, Beijing, 2002.

[SaU] J. Sacks and K. Uhlenbeck, The existence of minimal immersions of 2-spheres, Ann. of Math. 113 (1981), 1-24.

[ScU] Schoen, Richard; Uhlenbeck, Karen "Boundary regularity and the Dirichlet problem for harmonic maps." J. Differential Geom. 18 (1983), no. 2, 253268.

[Sim] Leon Simon, Lectures on Geometric Measure Theory, Australian National University Centre for Mathematical Analysis, Canberra, 1983

[Si] Simon, Leon "Existence of surfaces minimizing the Willmore functional." Comm. Anal. Geom. 1 (1993), no. 2, 281-326.

[St] M.Struwe "On the evolution of harmonic mappings of Riemannian surfaces" Comm. Math. Helvetici 60 (1985) 558-581.

[To] Toro, Tatiana Geometric conditions and existence of bi-Lipschitz parameterizations. Duke Math. J. 77 (1995), no. 1, 193227.

[Zhu] Zhu, Miaomiao Harmonic maps from degenerating Riemann surfaces. Math. Z. 264 (2010), no. 1, 6385 . 